Romeo Susan-Resiga

Professor

Hydraulic Machinery Department,

"Politehnica" University of Timişoara,

Bvd. Mihai Viteazu 1,

R0-300222, Timişoara, Romania

e-mail: resiga@mh.mec.utt.ro

Gabriel Dan Ciocan

Ecole Polytechnique Fédérale de Lausanne,

Laboratory for Hydraulic Machines,

Av. de Cour 33Bis, CH-1007,

Lausanne, Switzerland

e-mail: GabrielDan.Ciocan@epfl.ch

Ioan Anton

Professor

Member of the Romanian Academy

"Politehnica" University of Timişoara,

Hydraulic Machinery Department,

Bvd. Mihai Viteazu 1,

R0-300222, Timişoara, Romania

François Avellan

Professor

École Polytechnique Fédérale de Lausanne,

Laboratory for Hydraulic Machines,

Av. de Cour 33Bis, CH-1007,

Lausanne, Switzerland

e-mail: francois.avellan@epfl.ch

\section{Analysis of the Swirling Flow Downstream a Francis Turbine Runner}

An experimental and theoretical investigation of the flow at the outlet of a Francis turbine runner is carried out in order to elucidate the causes of a sudden drop in the draft tube pressure recovery coefficient at a discharge near the best efficiency operating point. Laser Doppler anemometry velocity measurements were performed for both axial and circumferential velocity components at the runner outlet. A suitable analytical representation of the swirling flow has been developed taking the discharge coefficient as independent variable. It is found that the investigated mean swirling flow can be accurately represented as a superposition of three distinct vortices. An eigenvalue analysis of the linearized equation for steady, axisymmetric, and inviscid swirling flow reveals that the swirl reaches a critical state precisely (within 1.3\%) at the discharge where the sudden variation in draft tube pressure recovery is observed. This is very useful for turbine design and optimization, where a suitable runner geometry should avoid such critical swirl configuration within the normal operating range. [DOI: 10.1115/1.2137341]

\section{Introduction}

Swirling flow behavior in various technical applications has long been an intensive subject of research. Usually swirl effects are seen as either the desired result of design or unavoidable, possibly unforseen, side effects [1]. However, the hydraulic turbine draft tube on one hand benefits from the swirl at the runner outlet in order to mitigate flow detachment in the cone, but on the other hand suffers from the flow instabilities leading to pressure fluctuations and ultimately to the draft tube surge.

The draft tube of a hydraulic turbine is the machine component where the flow exiting the runner is decelerated, thereby converting the excess of kinetic energy into static pressure. In the case of machine rehabilitation of an existing power plant, mostly only the runner and the guide vanes are currently modified. For economical and safety reasons, the spiral casing and the draft tube are seldom redesigned, even if these components present some undesirable behavior. However, the installation of an upgraded runner requires a reliable prediction of the flow in a compact draft tube in order to avoid the peculiar and undesirable efficiency curve from Fig. 1. The efficiency drop as the discharge is increased above the best efficiency point value is found to be related to a corresponding sudden variation in the draft tube pressure recovery coefficient at the same discharge. It is this phenomenon we address in this paper.

The obvious practical importance of predicting the complex flow downstream the turbine runner, in the draft tube, led to the FLINDT research project of Flow Investigation in Draft Tubes [2]. The main objective of this project was to investigate the flow in hydraulic turbines draft tubes, for a better understanding of the

Contributed by the Fluids Engineering Division of ASME for publication in the JouRnal of Fluids EngineERING. Manuscript received July 9, 2004; final manuscript received July 31, 2005. Review conducted by Joseph Katz. physics of these flows and to build up an extensive experimental data base describing a wide range of operating points which can provide a firm basis for the assessment of the CFD engineering practice in this component. The extensive experimental investigation of the draft tube flow has been complemented with threedimensional numerical flow simulations $[3,4]$ aimed at elucidating the swirling flow evolution up to the turbine outlet as well as the phenomena that led to the peculiar sudden drop in the turbine efficiency.

Other investigations have been mainly focused on the ability of the CFD tools to accurately reproduce the complex threedimensional velocity and pressure field in draft tubes for Kaplan turbines $[5,6]$. One important issue addressed in these studies was the sensitivity of numerical results to the boundary conditions, particularly the inlet ones.

The present paper focuses on the structure of the swirl produced by the constant pitch turbine runner and further ingested by the draft tube. The corresponding hydrodynamic field is a direct outcome of the runner design and the operating point. Since changing the runner design, while keeping the same draft tube, may lead to an unexpected sudden efficiency drop for a certain discharge, it would be preferable that some design criteria be put forward as far as the runner outlet swirl is concerned. The present analysis shapes such criteria by using relative simple mathematical and numerical tools. Of course, the complex three-dimensional and unsteady flow in the draft tube cannot be quantitatively predicted only by analyzing the draft tube inlet swirl. However, if the runner outlet swirl structure displays a sudden change with respect to appropriate criteria, and this change occurs at a discharge close to the experimental one where the sudden drop in turbine efficiency is observed, these criteria should be taken into account when designing or redesigning the runner.

In analyzing a swirling flow one benefits from a large body of literature on this subject. In laboratory investigations swirl was 


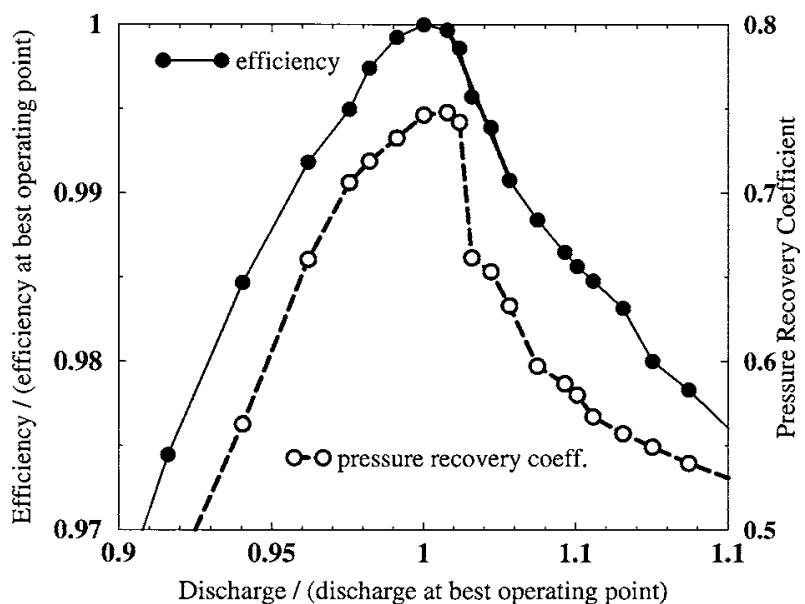

Fig. 1 Efficiency break off obtained by increasing the discharge and keeping the specific energy constant. Model test of a Francis turbine with specific speed 0.56 .

generated by adjustable guidevanes, by fixed vanes similar to those employed in combustors, or by tangential inflow through a long slit [1]. The closest setup to the hydraulic turbine case seems to be the adjustable radial guidevane apparatus, which has been largely used for more than 50 years to investigate, both experimentally and numerically, the so-called vortex breakdown (VB) phenomenon [7-10]. The formulas employed in these studies to fit both axial and circumferential velocity component radial variation are of particular relevance for our study.

Several theoretical developments have been devoted to explain the VB. However, a general consensus over the definition of this phenomenon has not been reached yet. For example, Benjamin [11] considers the VB to be a finite transition between two dynamically conjugate states of axisymmetric flow, analogous to the hydraulic jump in open-channel flow. A similar definition was later adopted by Keller [12], who argued that various authors or even schools have conflicting views on the correct interpretation of the physics of VB. Leibovich [13] relates VB to a disturbance characterized by the formation of an internal stagnation point on the vortex axis, followed by reversed flow in a region of limited axial extent. Goldshtik and Hussain [14] consider that VB occurs due to solution nonuniqueness in some range of inflow parameters when the entire steady flow experiences a jump to another metastable steady state with the same boundary conditions. They stress that VB is a loss-free process and, hence, analogies with shocks or hydraulic jumps are misleading and must be abandoned. All theories for confined swirling flows consider axisymmetric geometries with constant or variable cross section (e.g., slowly diverging pipes). It is difficult to imagine that a simplified theory could be elaborated for a swirling flow in an actual draft tube with both cross-section shape and area variation, as well as changes in the flow direction. However, at least for the draft tube cone where most of the pressure recovery occurs, swirling flow theories might provide valuable results for design evaluation and optimization.

Mauri et al. [15,3] developed and applied original techniques to analyze the three-dimensional flow in the FLINDT draft tube. They explain the draft tube efficiency drop from Fig. 1 by a global instability triggered by the flow rate increase. The topological structure of the velocity field changes abruptly with the emergence of a saddle point and a focus in the skin friction lines pattern on the elbow wall, leading to a global Werlé-Legendre separation that blocks the right channel. However, there is an important question to be answered: is this phenomenon the primary cause of the draft tube efficiency drop or it is one of the consequences of a corresponding abrupt change in the swirling flow ingested by the draft tube as the discharge increases? It is this question we address in

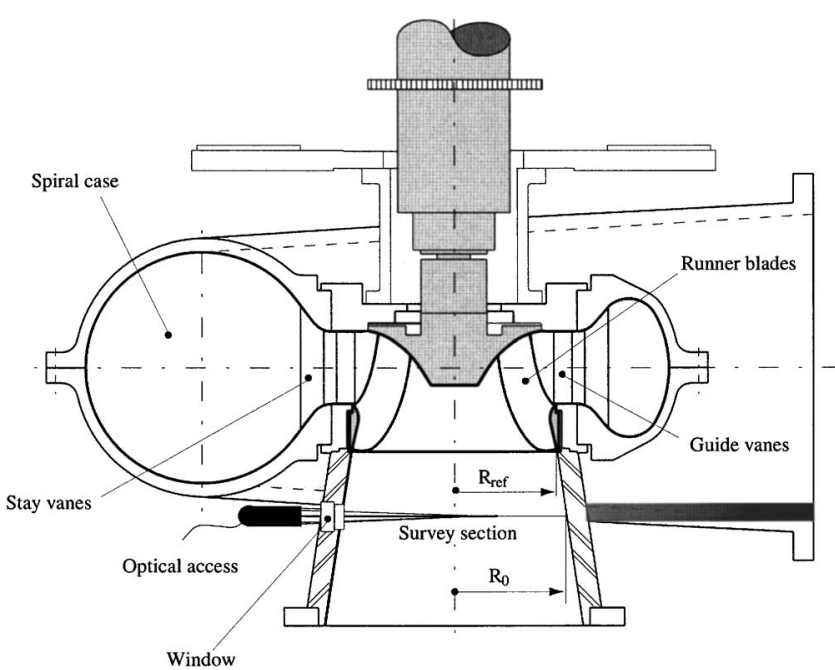

Fig. 2 Sketch of the Francis turbine model and LDA setup for the flow survey section at runner outlet-draft tube inlet

this paper, by investigating the swirling flow on the draft tube inlet section within the general framework of vortex breakdown theories.

In Sec. 2 we briefly present the experimental setup and measuring techniques used to investigate the flow in a Francis turbine draft tube. In this paper we examine the flow on a section at the runner outlet/draft tube inlet. Laser Doppler anemometry has been employed to investigate the velocity components, with particular attention paid to the data error control.

Section 3 is devoted to the analytical representation of velocity components radial variation. A critical analysis of swirling flow models available in literature is followed by the development of a model particularly suited to hydraulic turbines. It is shown that a three-vortex system accurately represents the experimental data, and a least squares technique is employed for computing the model parameters. Finally, the velocity profiles are parametrized only by the discharge coefficient, thus allowing a swirl behavior analysis as the operating point changes continuously.

The nonlinear Long-Squire equation is used in Sec. 4 as a mathematical model for the swirling flow at the draft tube inlet. The finite element method is employed to solve the corresponding boundary value problem for the stream function.

The solution behavior is examined in Sec. 5 using the linearized operator spectrum analysis. It was found that the critical state of the swirl configuration, defined by Benjamin [11], is in good agreement with the abrupt change experimentally observed in the draft tube pressure recovery coefficient.

The paper conclusions are summarized in Sec. 6 .

\section{Experimental Investigation of the Velocity Field on the Draft Tube Inlet}

The FLINDT project [2] experimental investigations were carried out on a Francis turbine scaled model of specific speed 0.56 (Fig. 2). The turbine model has a spiral casing of double curvature type with a stay ring of 10 stay vanes, a distributor made of 20 guide vanes, a 17-blade runner of a $0.4 \mathrm{~m}$ outlet diameter, and a symmetric elbow draft tube with one pier. The global measurements for flow rate, head, and efficiency were performed according to the IEC 60193 International Standard [16].

The experimental data used in this paper were obtained with a two-component probe Laser Doppler Anemometer (LDA), using back-scattered light and transmission by optical fiber, with a laser of $5 \mathrm{~W}$ argon-ion source. The main characteristics of the optical system are laser wave lengths $488 / 514.5 \mathrm{~nm}$, probe diameter 


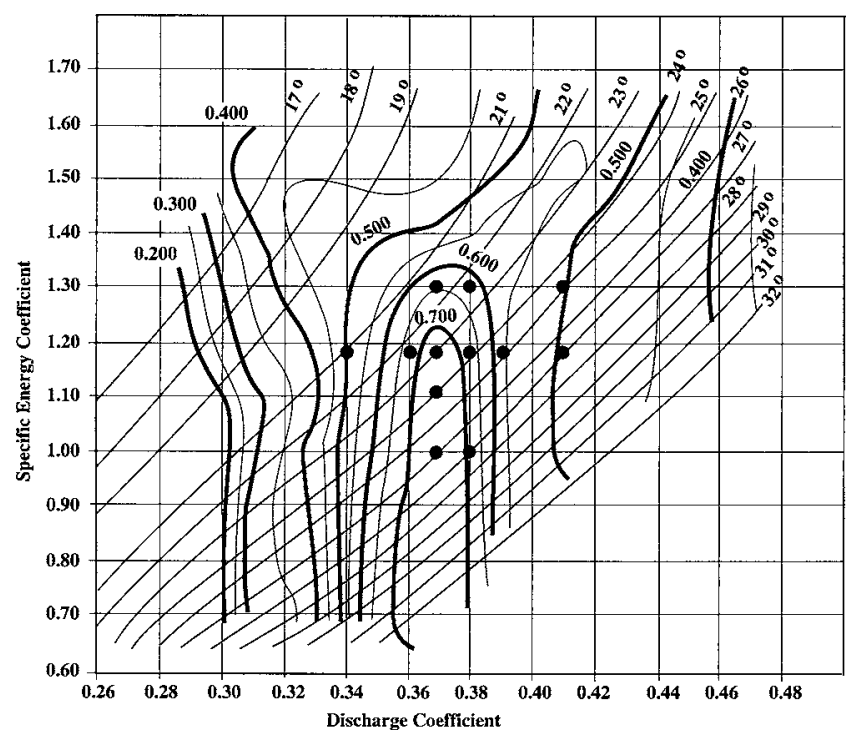

Fig. 3 Pressure recovery isolines (thick lines) for the draft tube investigated in the FLINDT project. The turbine operating points (discharge coefficient-specific energy coefficient) are shown with filled circles.

$60 \mathrm{~mm}$, beam spacing probe with beam expander $73.3 \mathrm{~mm}$, focal length $1000 \mathrm{~mm}$, fringe spacing $\sim 5.3 \mathrm{~nm}$, and measuring volume $\sigma_{x}=\sigma_{y} \sim 0.2 \mathrm{~mm}, \sigma_{z} \sim 6 \mathrm{~mm}$.

Spherical silver-coated glass particles are introduced in the test rig flow. These particles are hollowed in order to match the water density and are able to follow flow fluctuations frequency up to $5 \mathrm{kHz}$ [17]. The mean diameter of these particles is $10 \mu \mathrm{m}$.

In order to control the position of the measurement volume, a ray tracing technique is used for calculating direct and inverse light paths of laser beams through the different media (air, window, water). An optical window with plane and parallel faces is used as an interface. The measuring point geometrical location is controlled within a $0.05 \mathrm{~mm}$ accuracy. Both axial and circumferential components of the velocity are measured. The uncertainties of the velocity measurements are estimated to be $2 \%$ of the measured value [18].

The global "efficiency" of the draft tube is quantified using the static pressure recovery coefficient, defined as

$$
\chi=\frac{(p / \rho+g z)_{o u t}-(p / \rho+g z)_{r e f}}{Q^{2} / 2 A_{r e f}^{2}} .
$$

Figure 3 presents isolines of the pressure recovery coefficient in discharge coefficient-energy coefficient coordinates. The operating points further referenced in this paper, where full velocity measurements are performed on the survey section from Fig. 2, are also marked. The turbine efficiency break-off, Fig. 1, is found to be produced by a corresponding drop in the draft tube pressure recovery. This phenomenon occurs practically at the same discharge value for a specific energy coefficient lower than 1.30.

Throughout this paper the velocity is made dimensionless by the runner angular speed $\times$ runner outlet radius, and lengths are made dimensionless with respect to the runner outlet radius $R_{\text {ref }}$ (Fig. 2)

In order to assess the Reynolds number influence on the velocity field at the runner outlet, the same operating point (discharge coefficient, specific energy coefficient) has been investigated for two runner rotational speed values, 500 and $1000 \mathrm{rpm}$, respectively. The data for dimensionless axial and circumferential velocity components corresponding to the same operating point in Fig. 3 but at two runner rotational speeds are plotted in Fig. 4. According to the IEC 60193 Standard [16], the characteristic Reynolds number $\operatorname{Re}$ of the turbine is defined as $\operatorname{Re}=U D / \nu=\pi n D^{2} / 60 \nu$.

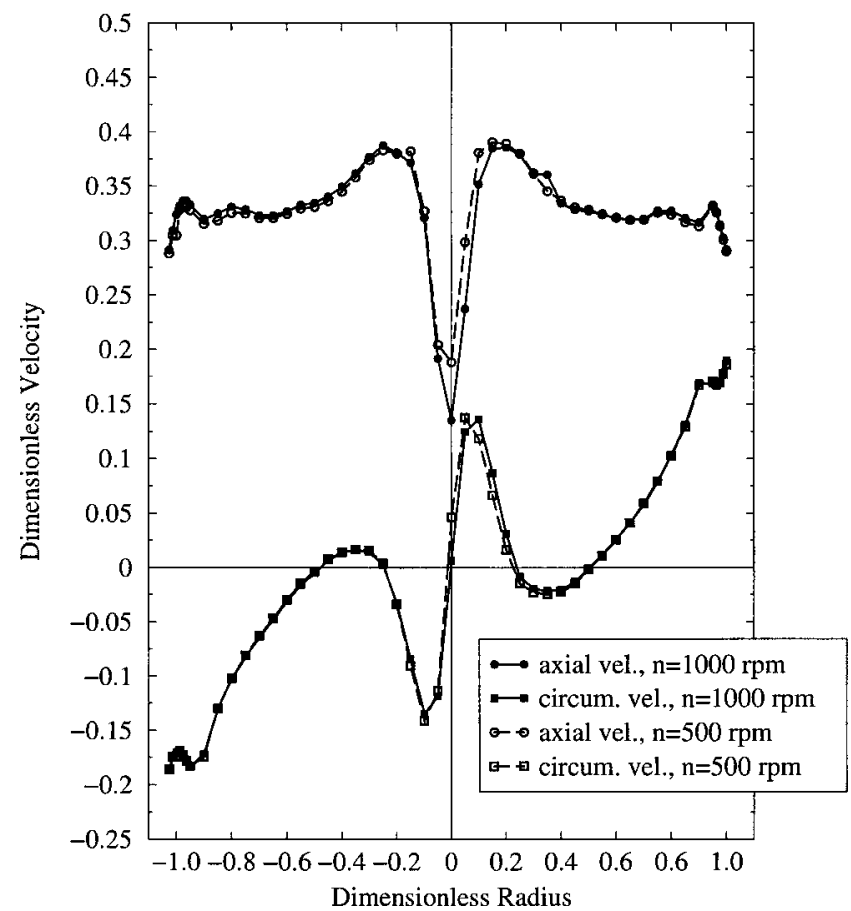

Fig. 4 Reynolds number influence on the dimensionless velocity profiles at operating point with discharge coefficient 0.368 and energy coefficient 1.18

The corresponding Reynolds number of the reduced scale model is changed from $4.2 \times 10^{6}$ (at $n=500 \mathrm{rpm}$ ) to $8.4 \times 10^{6}$ (at $n$ $=1000 \mathrm{rpm}$ ) without any significant variation in the dimensionless velocity profiles. Moreover, the axial and circumferential velocity profiles measured at the same discharge coefficient value are not sensitive to specific energy coefficient changes within the investigated range 1.0-1.3, as one can observe Fig. 5. This led us to the conclusion that the only relevant parameter for the investigation further presented in this paper is the turbine discharge coefficient.

\section{Analytical Representation of Axial and Circumfer- ential Velocity Profiles}

Several swirling flow models have been considered in the literature to study either the vortex stability or the vortex breakdown. We briefly review these models in order to develop a suitable representation for the swirl at the Francis runner outlet. Historically, vortex flow have been first studied in unbounded media and as a result the velocity circulation at very large distance from the vortex axis was naturally chosen as a vortex parameter. Since we are dealing with confined vortices, it is convenient to use the angular velocity at the vortex axis, $\Omega$. A second parameter is a characteristic vortex radius $R$ which measures the vortex core radial extent. These two parameters define the Rankine vortex circumferential velocity,

$$
w(r)=\left\{\begin{array}{cc}
\frac{\Omega R^{2}}{r} & \text { for } r \geqslant R, \\
\Omega r & \text { for } r<R
\end{array},\right.
$$

where $r$ is the radial distance from the vortex axis. This simplified model provides a continuous function for $w(r)$, but the derivative is discontinuous. A rigorous theoretical foundation is provided for the Burgers vortex (also known as the Lamb vortex), which gives the circumferential velocity profile as 


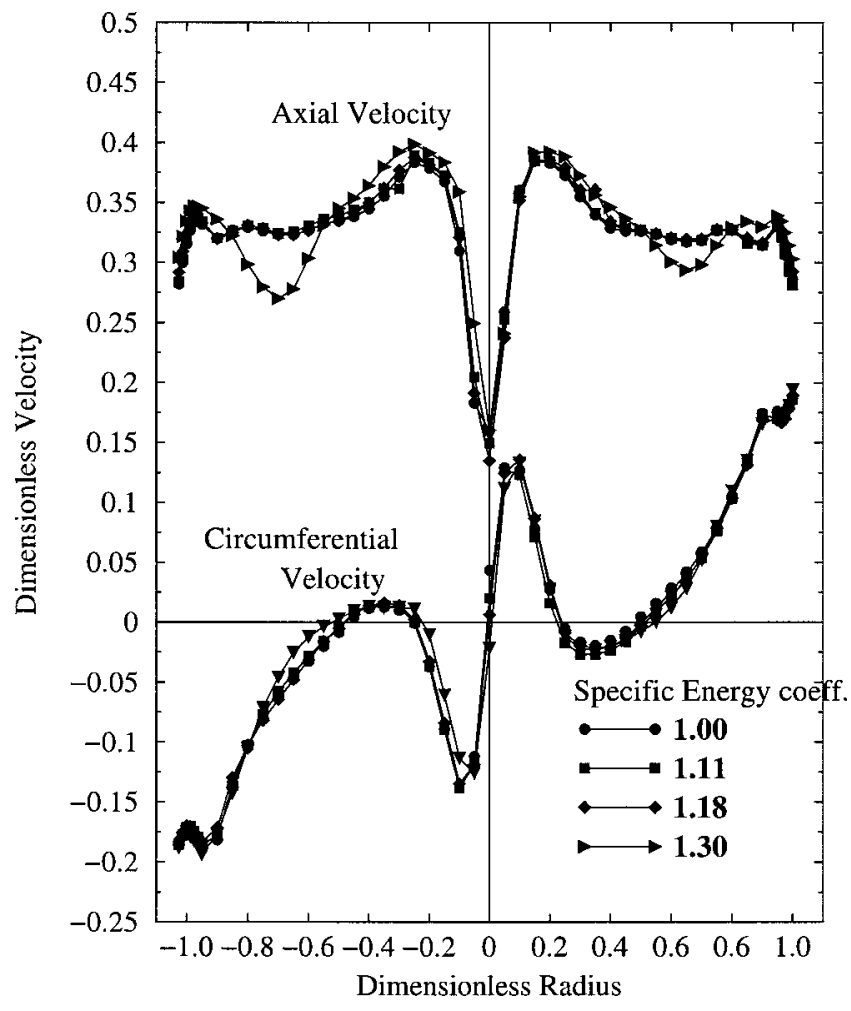

Fig. 5 Specific energy coefficient influence on the dimensionless velocity profiles at operating points with discharge coefficient $\mathbf{0 . 3 6 8}$

$$
w(r)=\frac{\Omega R^{2}}{r}\left[1-\exp \left(-\frac{r^{2}}{R^{2}}\right)\right] .
$$

Formula (3) is an exact solution for a viscous vortex produced by radial inflow and axial outflow where the conditions at large radial distance are irrotational. The relationship between Rankine vortex (2) and Burgers vortex (3) models can be easily seen from Fig. 6. If we take the limit for $r \ll R$ in (3) we get $\Omega r$, while for $r \gg R$ we obtain $\Omega R^{2} / r$. In conclusion, the Rankine vortex represents the asymptotic behavior of the Burgers vortex for large and small radius with respect to the vortex core extent $R$.

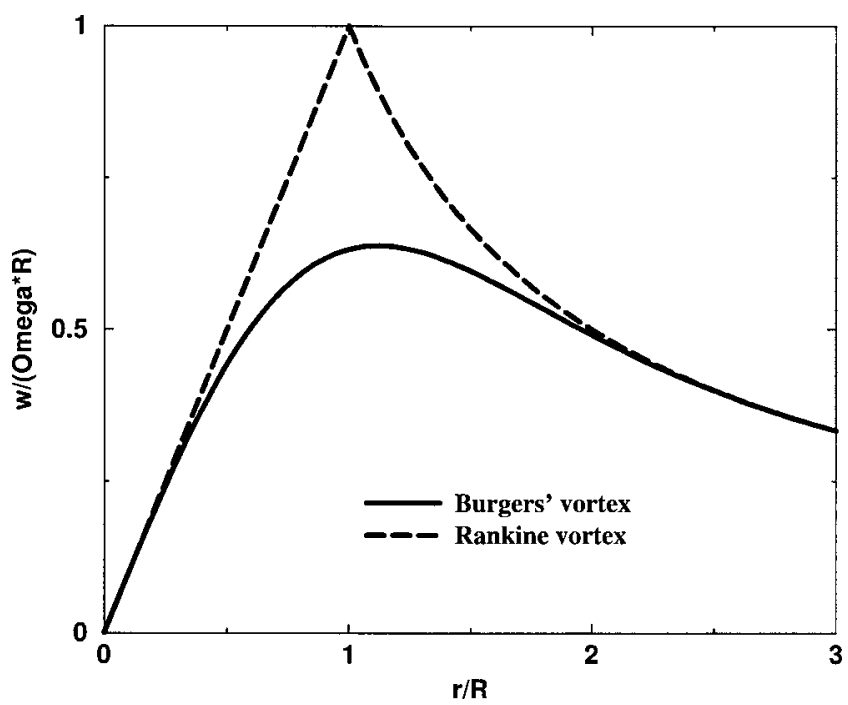

Fig. 6 Circumferential velocity profile for Rankine and Burgers vortex models, respectively
Both the above models consider a uniform axial velocity. It was Batchelor [19] who pointed out that a radial variation in circumferential velocity must be accompanied by a variation in the axial velocity. He showed that in the case of a trailing vortex from one side of a wing in an infinite body of fluid all streamlines originate in a region where the pressure is uniform and the fluid velocity is uniform with only an axial component $U_{0}$. When a Rankine vortex circumferential velocity (2) is induced by viscous effects in the boundary layer of the wing, the axial velocity inside the vortex core increases as

$$
u= \begin{cases}U_{0} & \text { for } r \geqslant R, \\ \sqrt{U_{0}^{2}+2 \Omega^{2}\left(R^{2}-r^{2}\right)} & \text { for } r<R,\end{cases}
$$

Applying the same considerations for the Burgers vortex (3), we obtain

$$
u^{2}=U_{0}^{2}+\int_{r}^{\infty} \frac{1}{r^{2}} \frac{\partial K^{2}}{\partial r} d r=U_{0}^{2}+2 \Omega^{2} R^{2}\left[\operatorname{Ei}_{1}\left(\frac{r^{2}}{R^{2}}\right)-\operatorname{Ei}_{1}\left(2 \frac{r^{2}}{R^{2}}\right)\right],
$$

where $K \equiv r w$ is $(2 \pi)^{-1}$ times the circulation around a symmetrically placed circle and $\mathrm{Ei}_{1}$ is the exponential integral of order one. On the axis the axial velocity is $\sqrt{U_{0}^{2}+2 \ln (2) \Omega^{2} R^{2}}$, which is smaller than the corresponding value for the Rankine vortex $\sqrt{U_{0}^{2}+2 \Omega^{2} R^{2}}$.

Faller and Leibovich [8] have used the following axial velocity functional form to fit their experimental data for a radial guidevane swirl generator,

$$
u(r)=U_{0}+U_{1} \exp \left(-\frac{r^{2}}{R^{2}}\right),
$$

where $U_{1}$ is the difference between the axial velocity on the axis and the axial velocity far away from the axis, $U_{0}$. Note that when using (5) together with (3) the vortex core radius $R$ is the same. When $R \gg r$, Eq. (3) becomes $u(r) \approx \Omega r$ since $\lim _{x \rightarrow 0}[1$ $\left.-\exp \left(-x^{2}\right)\right] / x^{2}=1$, and Eq. (5) becomes $u(r) \approx U_{0}+U_{1}=$ const.

It was specifically stated in [8] that no theoretical justification for (5) is available. Indeed, in comparison with the axial velocity profile obtained, according to Batchelor, within the constant total head hypothesis

$$
\frac{u(r)}{U_{0}}=\sqrt{1+2\left(\frac{\Omega R}{U_{0}}\right)^{2}\left[\operatorname{Ei}_{1}\left(\frac{r^{2}}{R^{2}}\right)-\operatorname{Ei}_{1}\left(2 \frac{r^{2}}{R^{2}}\right)\right]},
$$

the functional form (5) rewritten to have the same axial velocity

$$
\frac{u(r)}{U_{0}}=1+\left[-1+\sqrt{1+2 \ln (2)\left(\frac{\Omega R}{U_{0}}\right)^{2}}\right] \exp \left(-\frac{r^{2}}{R^{2}}\right),
$$

seems to be completely different. However, one can easily conclude from Fig. 7 that $(6 b)$ is a rather good approximation for $(6 a)$. Obviously, $(6 b)$ or the more general form (5) is more convenient for analytical manipulation.

A more rigorous justification for (5) is attempted by Alekseenko et al. [20] who consider swirling flows with helical symmetry, i.e., the flow characteristics conserve their values along helical lines of pitch $2 \pi l$. For axisymmetrical (columnar) helical vortices with a circumferential velocity as in (3) they obtain the axial velocity profile of the form

$$
u(r)=U_{\text {axis }}-\frac{\Omega R^{2}}{l}\left[1-\exp \left(-\frac{r^{2}}{R^{2}}\right)\right],
$$

where $U_{\text {axis }} \equiv U_{0}+U_{1}$. One can identify from (7) the characteristic velocity $U_{1}=\Omega R^{2} / l$, and eventually use the length $l=(2 \pi)^{-1}$ $\times$ pitch instead of $U_{1}$ as a free parameter.

So far we have considered only an elementary vortex representation. However, the experimental data display a more complex structure which should be modeled by a combination of simple vortices. There are two possibilities to consider such combinations. One idea put forward by Alekseenko et al. [20] is to con- 


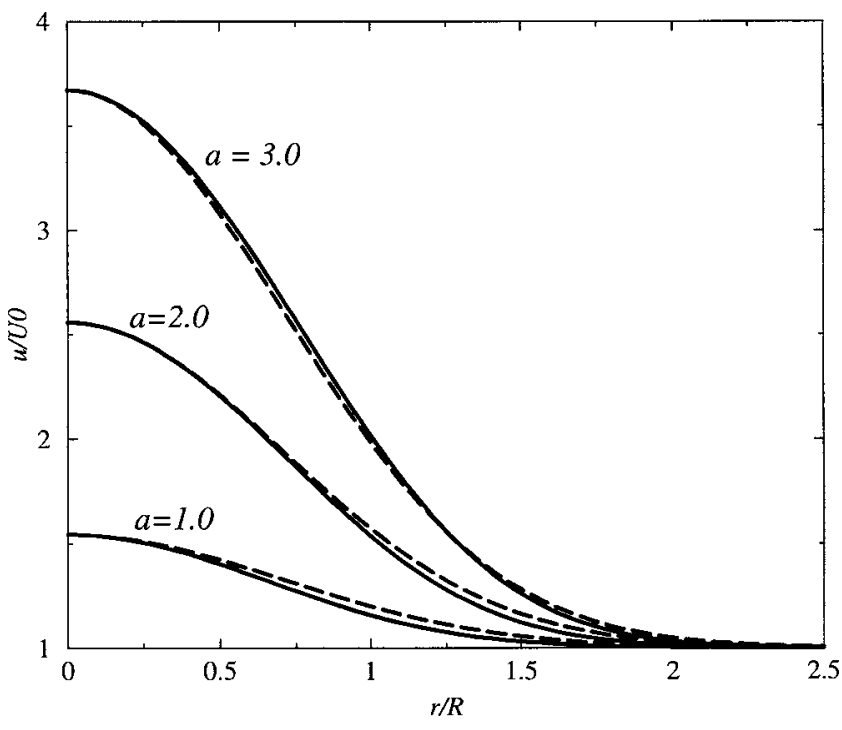

Fig. 7 Axial velocity profiles computed with (6a)-solid lines and $(6 b)$-dashed lines, respectively, for several values of the dimensionless parameter $a \equiv \Omega R / U_{0}$

sider nonoverlapping regions along the radius, with piecewise continuous vorticity distribution. The resulting velocity profiles (both axial and circumferential) are made continuous by a proper choice of the integration constants. Another idea was put forward by Mattner et al. [9] who considered a sum of elementary velocity profiles for both axial and circumferential components. Essentially this second approach becomes equivalent to the first one if the vortices are well separated, i.e., the characteristic radii are well distinct one from each other.

In order to build a suitable vortex combination we should first consider a base flow. Using the dimensionless velocity components $u$ and $w$, as well as the dimensionless runner tangential velocity, which coincides with the dimensionless radius $r$ according to Sec. 2, the relative flow angle is

$$
\beta=\arctan \frac{u}{r-w} .
$$

Since the swirling flow examined in this paper is produced by a constant pitch Francis turbine runner, the relative flow angle should be consistent with an approximation corresponding to a solid body rotation, $w=\Omega_{0} r$ and $u=U_{0}$. Indeed, the relative flow angle computed from the experimental data for circumferential and axial velocity can be reasonably fitted with $\beta$ $=\arctan ($ const $/ r)$, as shown in Fig. 8. However, a solid body rotation is a rather crude approximation of the actual velocity profiles. Figures 4 and 5 suggest that two Batchelor vortices, one co-rotating and the other counter-rotating with respect to $w=\Omega_{0} r$, and co-flowing/counter-flowing with respect to $u=U_{0}$, respectively, should be superimposed for consistency with experimental data for circumferential and axial velocity profiles:

$$
\begin{gathered}
w(r)=\Omega_{0} r+\Omega_{1} \frac{R_{1}^{2}}{r}\left[1-\exp \left(-\frac{r^{2}}{R_{1}^{2}}\right)\right]+\Omega_{2} \frac{R_{2}^{2}}{r}\left[1-\exp \left(-\frac{r^{2}}{R_{2}^{2}}\right)\right], \\
u(r)=U_{0}+U_{1} \exp \left(-\frac{r^{2}}{R_{1}^{2}}\right)+U_{2} \exp \left(-\frac{r^{2}}{R_{2}^{2}}\right) .
\end{gathered}
$$

If $R_{0}$ is the dimensionless survey section radius, then the discharge coefficient can be obtained by integrating the axial velocity profile $(9 b)$,

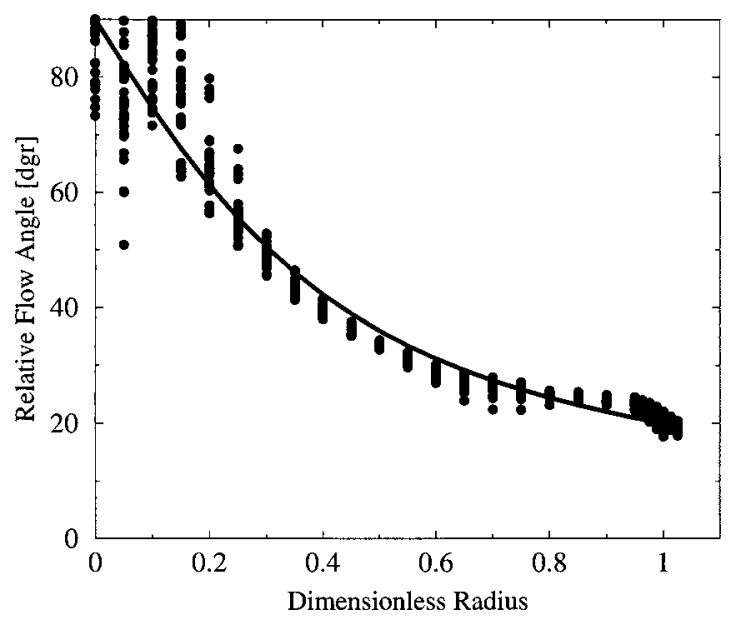

Fig. 8 Relative flow angle computed from the experimental data for axial and circumferential velocity components on the survey section. The solid curve is a least squares fit considering a rigid body rotation for the circumferential velocity and a constant axial velocity.

$$
\varphi=U_{0} R_{0}^{2}+U_{1} R_{1}^{2}\left[1-\exp \left(-\frac{R_{0}^{2}}{R_{1}^{2}}\right)\right]+U_{2} R_{2}^{2}\left[1-\exp \left(-\frac{R_{0}^{2}}{R_{2}^{2}}\right)\right] .
$$

The functional forms (9) have an eight-parameter set $\mathbf{\Pi}$ $\equiv\left\{R_{1}, R_{2}, \Omega_{0}, \Omega_{1}, \Omega_{2}, U_{0}, U_{1}, U_{2}\right\}$ to be determined by fitting the experimental data. For each operating point under consideration, with a set of experimental data $\left(r_{j}, u_{j}, w_{j}\right) j=1, \ldots, N$, the error vector $\mathbf{e}(\boldsymbol{\Pi})=\left\{e_{k}(\boldsymbol{\Pi})\right\}, k=1,2, \ldots, 2 N$ is defined as

$$
e_{k}(\boldsymbol{\Pi})= \begin{cases}u\left(r_{k}, \boldsymbol{\Pi}\right)-u_{k} & \text { for } k=1,2, \ldots, N, \\ w\left(r_{k-N}, \boldsymbol{\Pi}\right)-w_{k-N} & \text { for } k=N+1, \ldots, 2 N .\end{cases}
$$

The error vector includes both axial and circumferential velocity data since the vortex core radii $R_{1}$ and $R_{2}$ correspond to both velocity components. The parameter set is found by minimizing $\sum_{k=1}^{2 N}\left[e_{k}(\boldsymbol{\Pi})\right]^{2}$, leading to a least squares estimate of $\boldsymbol{\Pi}$. Let $\boldsymbol{\Pi}_{c}$ be the current estimate of $\boldsymbol{\Pi}$. A new estimate is given by $\boldsymbol{\Pi}_{c}+\boldsymbol{\Pi}_{c}^{*}$, where $\boldsymbol{\Pi}_{c}^{*}$ is a solution to

$$
\left[\mathbf{J}^{T}\left(\boldsymbol{\Pi}_{c}\right) \mathbf{J}\left(\boldsymbol{\Pi}_{c}\right)+\mu_{c} \mathbf{I}\right] \boldsymbol{\Pi}_{c}^{*}=\mathbf{J}^{T}\left(\boldsymbol{\Pi}_{c}\right) \mathbf{e}\left(\boldsymbol{\Pi}_{c}\right) .
$$

Here $\mathbf{J}\left(\boldsymbol{\Pi}_{c}\right)$ is the Jacobian $(2 N) \times 8$ matrix evaluated analytically at $\boldsymbol{\Pi}_{c}$. The iterative algorithm uses a "trust region" approach with a step bound of $\delta_{c}$. A solution of Eqs. (12) is first obtained for $\mu_{c}=0$. If $\left\|\boldsymbol{\Pi}_{c}^{*}\right\|^{2}<\delta_{c}$ this update is accepted. Otherwise, $\mu_{c}$ is set to a positive value and another solution is obtained.

Swirl parameters found by fitting formulas (9) to experimental data for 17 operating points are listed in Table 1. The last two columns contain the values of the discharge coefficient $\varphi$ computed with (10), and the corresponding relative error with respect to the measured value shown in the first column. This error is a good indicator for the accuracy of the fit, as well as for the measurements overall accuracy. We conclude that $(9 b)$ is a very good representation for the axial velocity at the runner outlet and the superposition of three vortices in $(9 a)$ accurately represents the experimental data for the circumferential velocity over the whole discharge range under investigation.

Figures 9-14 display the data as well as the curves fitted with (9) for the first six points in Table 1. These operating points cover the investigated discharge domain at a constant head corresponding to the turbine best efficiency operating point. The quality of the fit can be assessed by observing that most of the time the curves approach the experimental points within the measurement 
Table 1 Swirl parameters from Eqs. (9) for 17 turbine operating points

\begin{tabular}{|c|c|c|c|c|c|c|c|c|c|c|c|c|}
\hline \multicolumn{3}{|c|}{ Operating point } & \multicolumn{8}{|c|}{ Swirl parameters } & \multicolumn{2}{|c|}{ Discharge coefficient } \\
\hline $\begin{array}{l}\text { Discharge } \\
\text { coefficient }\end{array}$ & $\begin{array}{c}\text { Energy } \\
\text { coefficient }\end{array}$ & $\begin{array}{l}\text { Speed } \\
(\mathrm{rpm})\end{array}$ & $\Omega_{0}$ & $\Omega_{1}$ & $\Omega_{2}$ & $U_{0}$ & $U_{1}$ & $U_{2}$ & $R_{1}$ & $R_{2}$ & $\begin{array}{l}\text { Computed } \\
\text { Eq. (10) }\end{array}$ & Error \\
\hline 0.340 & 1.18 & 1000 & 0.31765 & -0.62888 & 2.2545 & 0.30697 & 0.01056 & -0.31889 & 0.46643 & 0.13051 & 0.344 & $+1.1 \%$ \\
\hline 0.360 & 1.18 & 1000 & 0.26675 & -0.79994 & 3.3512 & 0.31501 & 0.07324 & -0.29672 & 0.36339 & 0.09304 & 0.363 & $+0.8 \%$ \\
\hline 0.368 & 1.18 & 1000 & 0.27113 & -0.80310 & 3.4960 & 0.31991 & 0.08710 & -0.27350 & 0.37291 & 0.08305 & 0.372 & $+1.0 \%$ \\
\hline 0.380 & 1.18 & 1000 & 0.27536 & -0.81730 & 3.5187 & 0.32447 & 0.10618 & -0.23545 & 0.38125 & 0.07188 & 0.381 & $+0.2 \%$ \\
\hline 0.390 & 1.18 & 1000 & 0.27419 & -0.86579 & 3.2687 & 0.32916 & 0.12677 & -0.19061 & 0.37819 & 0.06502 & 0.389 & $-0.2 \%$ \\
\hline 0.410 & 1.18 & 1000 & 0.28802 & -0.96687 & 1.4590 & 0.33623 & 0.19121 & -0.09215 & 0.39108 & 0.05012 & 0.409 & $-0.3 \%$ \\
\hline 0.368 & 1.00 & 1000 & 0.27710 & -0.77440 & 3.3913 & 0.31704 & 0.08107 & -0.24619 & 0.38128 & 0.08289 & 0.368 & $+0.1 \%$ \\
\hline 0.380 & 1.00 & 1000 & 0.26726 & -0.83772 & 3.1082 & 0.32442 & 0.11387 & -0.19284 & 0.35948 & 0.07312 & 0.380 & $+0.1 \%$ \\
\hline 0.370 & 1.11 & 1000 & 0.28119 & -0.77668 & 3.5520 & 0.31731 & 0.08308 & -0.25254 & 0.38947 & 0.07904 & 0.369 & $-0.1 \%$ \\
\hline 0.368 & 1.30 & 1000 & 0.29078 & -0.79348 & 3.4239 & 0.31599 & 0.10086 & -0.25499 & 0.39536 & 0.07939 & 0.371 & $+0.8 \%$ \\
\hline 0.380 & 1.30 & 1000 & 0.27618 & -0.85846 & 3.2696 & 0.32691 & 0.12280 & -0.19933 & 0.37413 & 0.06734 & 0.386 & $+1.5 \%$ \\
\hline 0.410 & 1.30 & 1000 & 0.27670 & -0.96571 & 2.2165 & 0.33816 & 0.17829 & -0.10984 & 0.37930 & 0.05021 & 0.407 & $-0.6 \%$ \\
\hline 0.370 & 1.11 & 500 & 0.27854 & -0.77371 & 3.4491 & 0.31685 & 0.09058 & -0.21118 & 0.38535 & 0.07827 & 0.370 & $+0.1 \%$ \\
\hline 0.340 & 1.18 & 500 & 0.29630 & -0.67299 & 2.7487 & 0.30509 & 0.02987 & -0.32612 & 0.41942 & 0.11679 & 0.345 & $+1.6 \%$ \\
\hline 0.368 & 1.18 & 500 & 0.27151 & -0.78970 & 3.5902 & 0.31617 & 0.09131 & -0.22465 & 0.37450 & 0.07914 & 0.369 & $+0.2 \%$ \\
\hline 0.380 & 1.18 & 500 & 0.27659 & -0.79568 & 3.3111 & 0.32135 & 0.11063 & -0.17502 & 0.38765 & 0.07002 & 0.379 & $-0.3 \%$ \\
\hline 0.410 & 1.18 & 500 & 0.28624 & -0.93559 & 0.76010 & 0.33243 & 0.19587 & -0.06119 & 0.39588 & 0.05147 & 0.406 & $-0.9 \%$ \\
\hline
\end{tabular}

errors of $2 \%$. The wall boundary layer is not correctly reproduced since the swirling flow model (9) was specifically built for an inviscid flow analysis.

A main goal of this paper is to find a suitable parametric representation for the swirling flow at the Francis runner outlet. Figure 15 shows the variation of vortex characteristic angular velocities with respect to $\varphi$. Linear least squares fits accurately represent $\Omega_{0}(\varphi)$ and $\Omega_{1}(\varphi)$, while for $\Omega_{2}(\varphi)$ a parabolic fit seems to be quite satisfactory. Moreover, one should note that $\Omega_{0}$ is almost constant over the investigated operating range. The variation of vortex characteristic axial velocities with respect to $\varphi$ is shown in

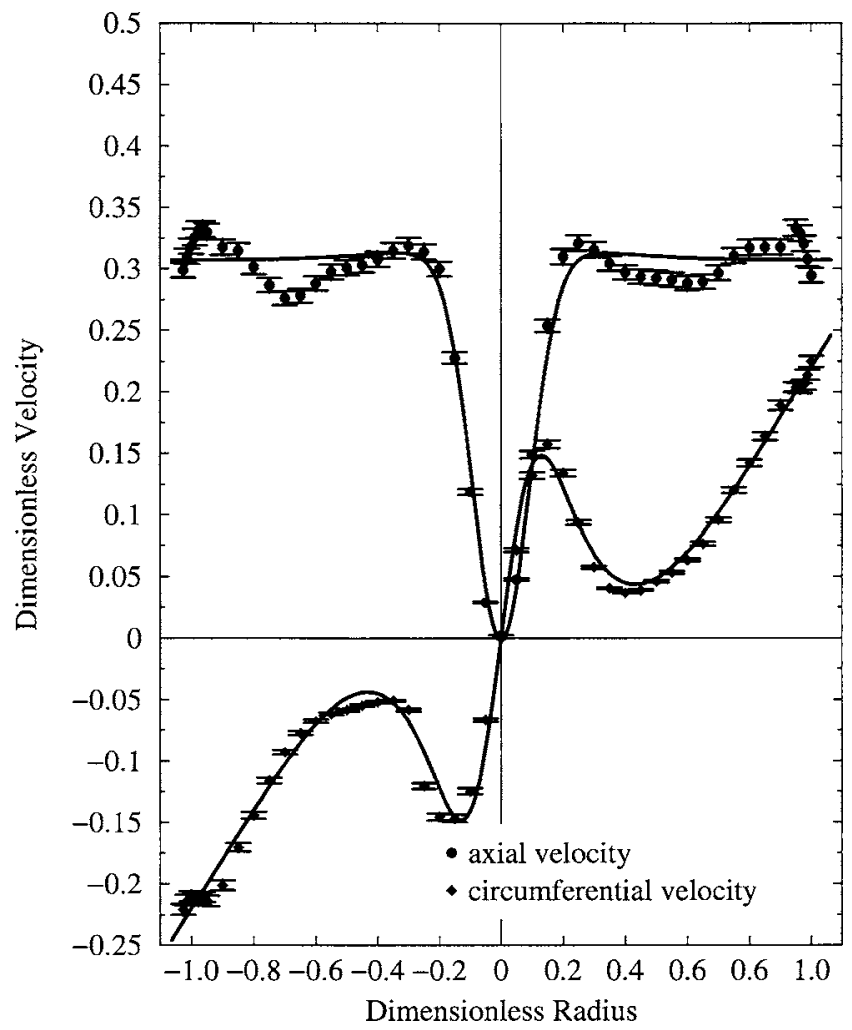

Fig. 9 Axial and circumferential velocity profiles at discharge $\varphi=0.340$
Fig. 16, together with the corresponding linear fits. Finally, Fig. 17 displays the dependence of the vortex core radii on $\varphi$. A first conclusion from Figs. 15-17 is that swirl parameters in (9) have a smooth, generally linear, variation in $\varphi$ over the investigated range. As a result, one obtains the velocity components as $C^{\infty}$ functionals $w(r, \varphi)$ and $u(r, \varphi)$, further employed in a parametric study of the flow stability or other properties.

According to the qualitative picture of the three vortex system presented in Table 2, Vortex 0 is a rigid body rotation with angular speed $\Omega_{0}$ and we can associate with it a constant axial velocity $U_{0}$. Vortex 1 , which has a vortex core extent about half the wall radius, is counter-rotating and co-flowing with respect to vortex 0 . The strength of this vortex, both in $\Omega_{1}$ as well as in $U_{1}$ is growing

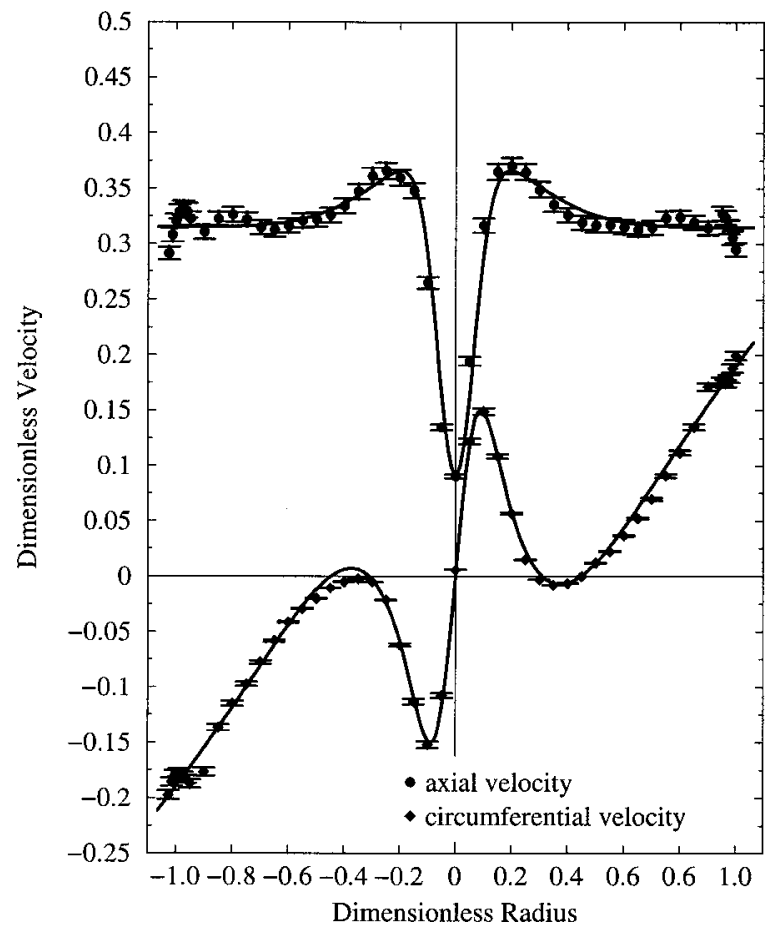

Fig. 10 Axial and circumferential velocity profiles at discharge $\varphi=0.360$ 


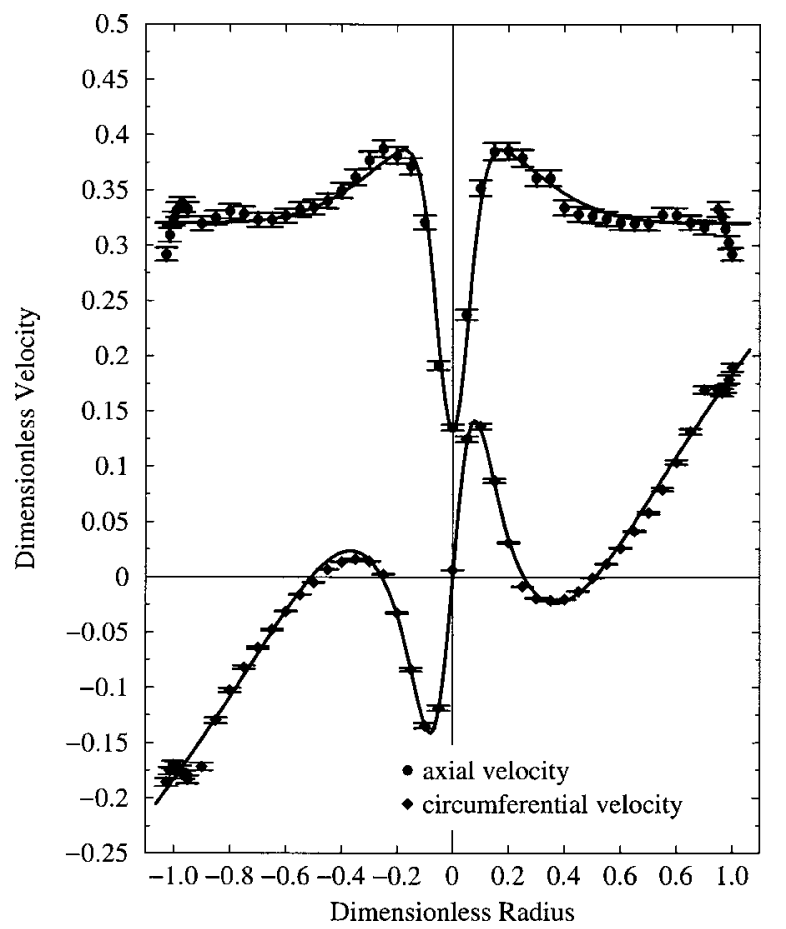

Fig. 11 Axial and circumferential velocity profiles at discharge $\varphi=0.368$

as the flow rate increases. Vortex 2 has a core at least four times smaller than vortex 1 , is co-rotating and counter-flowing with respect to vortex 0 , and its strength increases as the flow rate decreases. Note that as the flow rate increases (eventually beyond the upper limit in our investigation) vortex 2 will vanish. These two Batchelor vortices are mainly responsible for the swirling

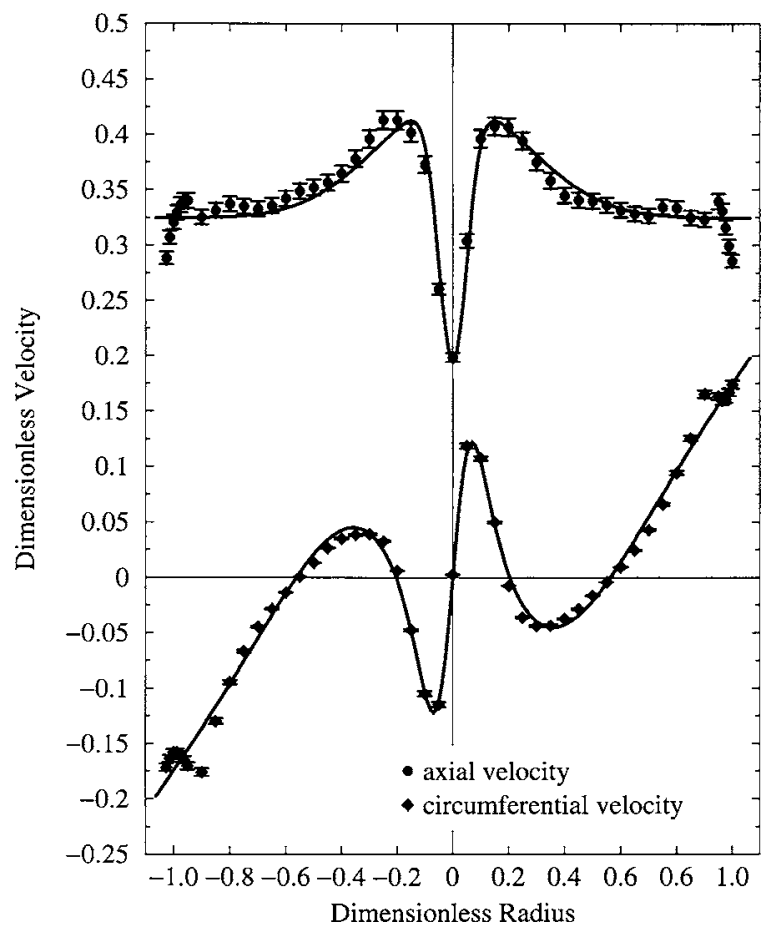

Fig. 12 Axial and circumferential velocity profiles at discharge $\varphi=0.380$

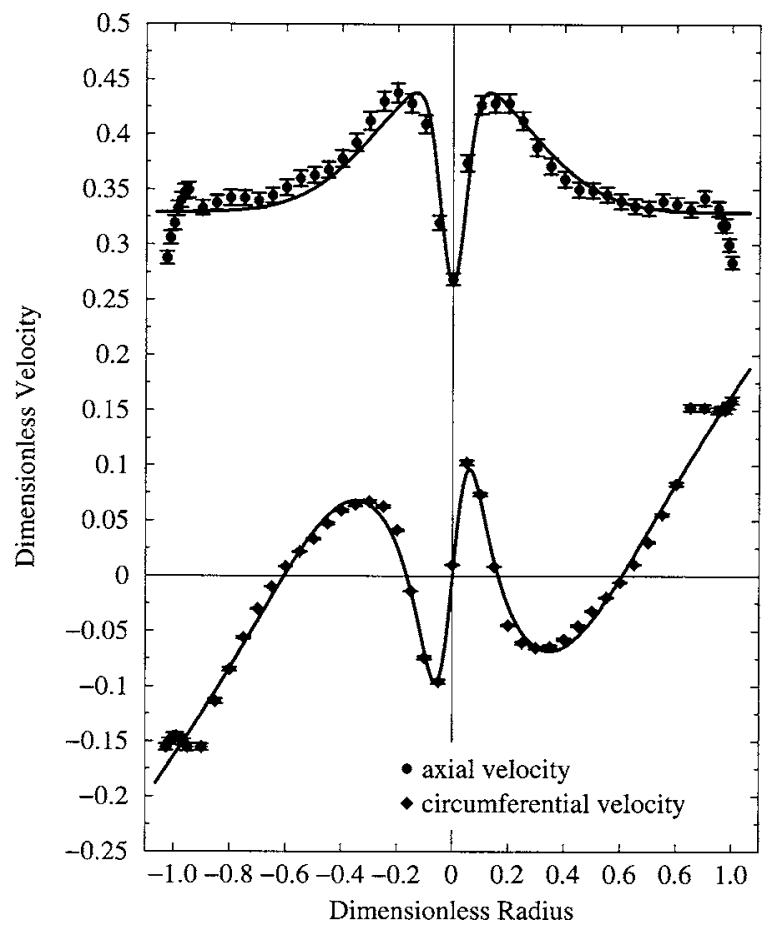

Fig. 13 Axial and circumferential velocity profiles at discharge $\varphi=0.390$

flow behavior. For $\varphi$ smaller than the design value a wake-like axial velocity is developed (Fig. 9) while for larger $\varphi$ the axial velocity has a jetlike profile (Fig. 14).

\section{Swirling Flow Mathematical Model and Numerical Approach}

Theoretical analysis of swirling flows can employ tools ranging from simplified axisymmetric, inviscid steady [11] or unsteady

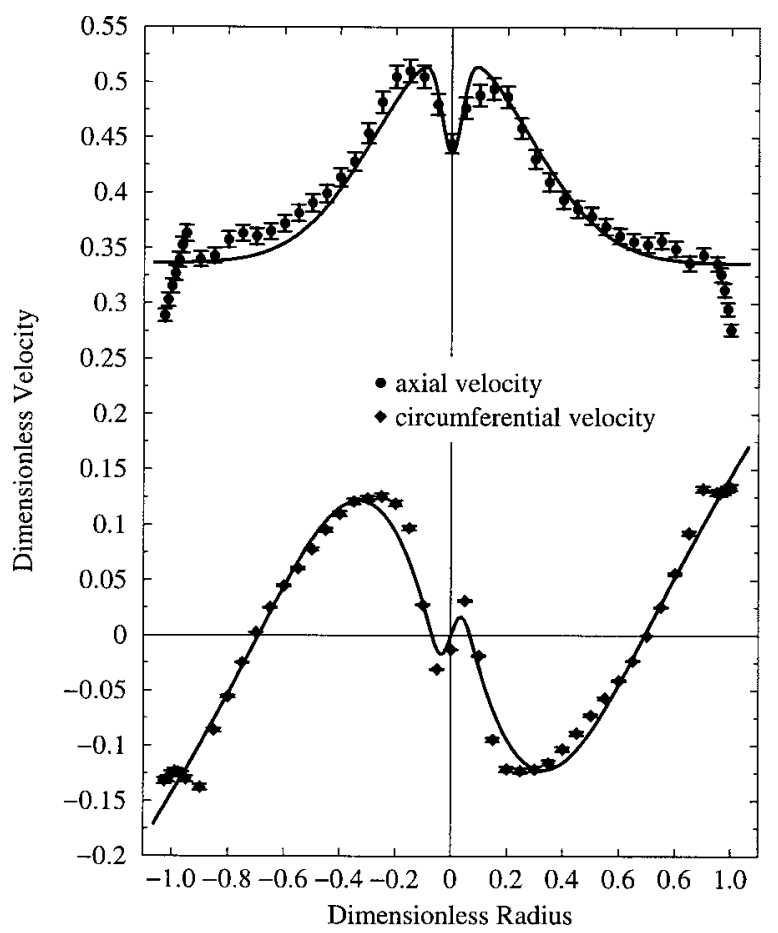

Fig. 14 Axial and circumferential velocity profiles at discharge $\varphi=0.410$ 


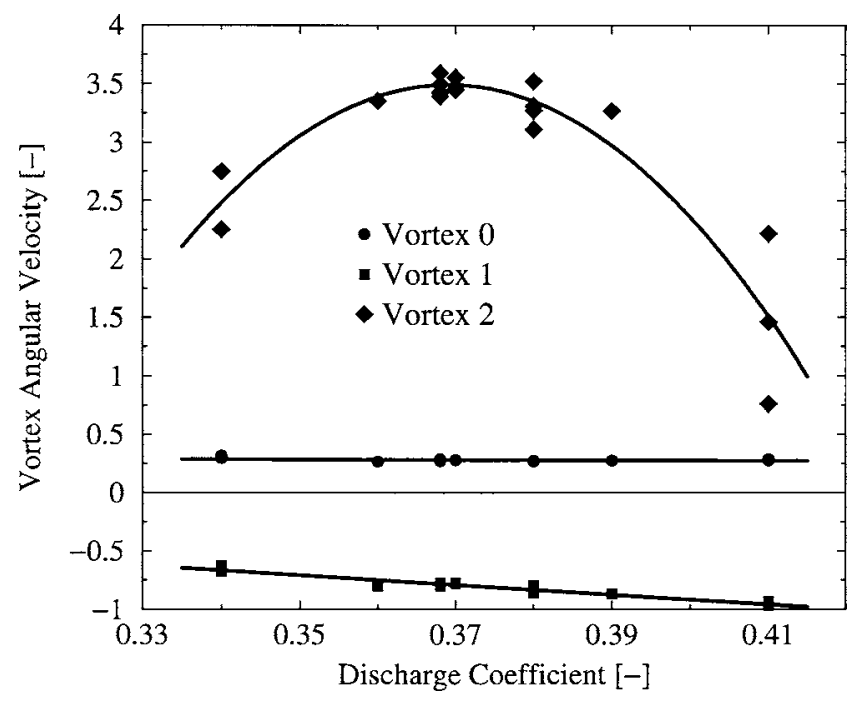

Fig. 15 Characteristic angular velocities $\Omega_{0}, \Omega_{1}$, and $\Omega_{2}$ versus discharge coefficient $\varphi$

[21] flow models to full 3D laminar [10] or turbulent [22] numerical simulation. However, it is useful to first examine the swirling flow at the draft tube inlet before performing an analysis of the flow in the straight cone or even in the whole 3D geometry. Such results may be quite useful if there is a correlation (even qualitative) with the overall draft tube behavior over a certain range of discharge variation.

If we restrict for now our analysis only for the runner outlet section, several simplifications must be admitted, and the results must be interpreted accordingly. We consider a steady mean flow with axial and circumferential velocity profiles derived from experimental data in Sec. 3. An inviscid incompressible fluid is considered, since our swirling flow representation does not account for the boundary layer near the wall.

The cylindrical flow assumption may not seem appropriate for the flow in the draft tube cone shown in Fig. 2, since it is known that the diverging pipe geometry precipitates the formation of breakdown by creating an adverse pressure gradient along the vortex axis. Shtern and Hussain [23] show that the nonparallel character of jets strongly affects their stability. Flow deceleration significantly enhances the shear-layer instability for both swirlfree and swirling jets. Buntine and Saffman [24] study a diverging flow using the steady axisymmetric Euler flow model. They show

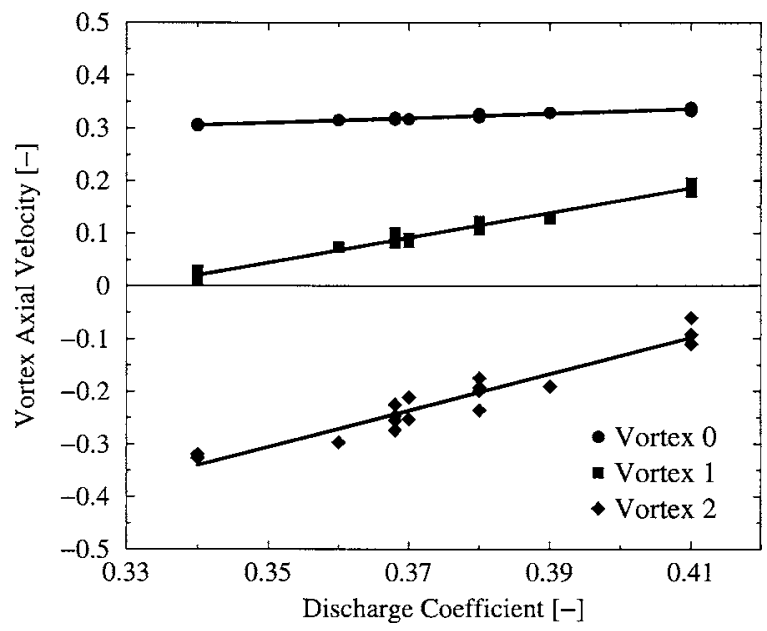

Fig. 16 Characteristic axial velocities $U_{0}, U_{1}$, and $U_{2}$ versus discharge coefficient $\varphi$

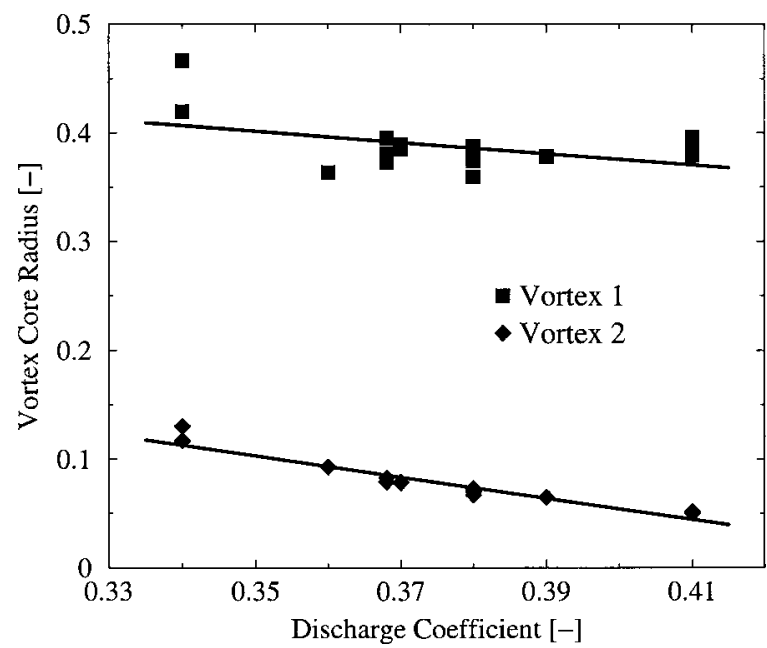

Fig. 17 Vortex core radii $R_{1}$ and $R_{2}$ versus discharge coefficient $\varphi$

that solutions fail to exist or, alternatively, that flow ceases to be unidirectional, so that the breakdown can be inferred, when a parameter measuring the relative magnitude of rotation and axial flow exceeds critical values depending upon geometry and inlet profiles. However, for slightly diverging duct of angles less than $2^{\circ}$, Tsai [25] shows that the flow can be considered locally parallel for the flow stability analysis. This parallel flow assumption is not quite restrictive even for the $8.5^{\circ}$ cone angle considered in this study. The diffusion process takes place only close to the wall, leading to a thin 3D boundary layer, as it can be seen from the measured velocity distribution (Figs. 4 and 5). Therefore, by neglecting the retarding influence of the wall, we can assume that the bulk flow is parallel. As far as the mean flow is concerned, the radial velocity is one order of magnitude smaller than the axial velocity since $v / u<\tan 8.5^{\circ} \approx 0.15$.

Within these assumptions, the mathematical model to be considered here corresponds to the theory of finite transitions between frictionless cylindrical flows originally developed by Benjamin [11]. The equation of continuity for axisymmetric incompressible flows is automatically satisfied by introducing the streamfunction $\psi(z, r)$ such that the axial and radial velocity components can be written as

$$
u=\frac{1}{r} \frac{\partial \psi}{\partial r} \quad \text { and } \quad v=-\frac{1}{r} \frac{\partial \psi}{\partial z}
$$

When applied to a circuit around a particular stream-surface $\psi$ =const Kelvin's theorem shows $r w$ to be a constant. Thus in general $r w \equiv K(\psi)$, where $K$ is a function of $\psi$ alone. Also, on a streamsurface the total specific energy $H=p / \rho+\left(u^{2}+v^{2}+w^{2}\right) / 2$ is constant by Bernoulli's theorem, thus $H$ is a function of $\psi$ alone. The momentum equation for the steady, axisymmetric swirling flow becomes

$$
\frac{1}{r^{2}}\left(\frac{\partial^{2} \psi}{\partial z^{2}}+\frac{\partial^{2} \psi}{\partial r^{2}}-\frac{1}{r} \frac{\partial \psi}{\partial r}\right)=H^{\prime}(\psi)-\frac{K(\psi) K^{\prime}(\psi)}{r^{2}},
$$

which is known in literature as the Long-Squire or BraggHawthorne equation. Goldshtik and Hussain [14] noted that, in fact, Eq. (14) was derived much earlier by Meissel (in 1873). The prime denotes differentiation with respect to $\psi$. By introducing the new variable $y=r^{2} / 2$ Eq. (14) can be rewritten as

$$
\frac{\partial^{2} \psi}{\partial y^{2}}+\frac{1}{2 y} \frac{\partial^{2} \psi}{\partial z^{2}}=H^{\prime}(\psi)-\frac{K(\psi) K^{\prime}(\psi)}{2 y} .
$$

If we substitute $Y_{1}=R_{1}^{2} / 2$ and $Y_{2}=R_{2}^{2} / 2$ the axial velocity profile $(9 b)$ can be written as 
Table 2 Swirling flow structure

\begin{tabular}{lccc}
\hline \hline & Vortex 0 & Vortex 1 & Vortex 2 \\
\hline Circumferential & rigid body rotation & counter-rotating & co-rotating \\
velocity & constant & co-flowing & counter-flowing \\
Axial velocity & - & $\sim 0.4 R_{0}$ & $\sim 0.1 R_{0}$ \\
Vortex core & & & \\
radius & & & \\
\hline \hline
\end{tabular}

$$
u(y)=U_{0}+U_{1} \exp \left(-y / Y_{1}\right)+U_{2} \exp \left(-y / Y_{2}\right) .
$$

Since from (13) $u=\partial \psi / \partial y$, the streamfunction for the above velocity profile is

$$
\psi(y)=U_{0} y+U_{1} Y_{1}\left[1-\exp \left(-y / Y_{1}\right)\right]+U_{2} Y_{2}\left[1-\exp \left(-y / Y_{2}\right)\right],
$$

where without loss of generality we have considered $\psi=0$ on the symmetry axis.

The discharge coefficient $\varphi$ from Eq. (10) can be rewritten as $\varphi=2 U_{0} Y_{0}+2 U_{1} Y_{1}\left[1-\exp \left(-Y_{0} / Y_{1}\right)\right]+2 U_{2} Y_{2}\left[1-\exp \left(-Y_{0} / Y_{2}\right)\right]$,

where $Y_{0}=R_{0}^{2} / 2$. As a result, at the wall we have $\psi_{\text {wall }} \equiv \psi(Y 0)$ $=\varphi / 2$.

For a mean flow with negligible radial velocity, the right-hand side in Eq. (15) is simply $d u / d y$,

$$
\begin{aligned}
H^{\prime}(\psi)-\frac{K(\psi) K^{\prime}(\psi)}{2 y}:= & \psi \stackrel{\text { Eq. } 17}{\longrightarrow} y \rightarrow-\frac{U_{1}}{Y_{1}} \exp \left(-\frac{y}{Y_{1}}\right) \\
& -\frac{U_{2}}{Y_{2}} \exp \left(-\frac{y}{Y_{2}}\right) .
\end{aligned}
$$

The map $\Psi \rightarrow y$ has to be computed numerically, for example using the Newton iterative method,

$$
y^{(m+1)}=y^{(m)}+\frac{\Psi-\psi\left(y^{(m)}\right)}{u\left(y^{(m)}\right)}, \quad \text { with initial guess } y^{(0)}=Y_{0} \frac{\Psi}{\varphi / 2},
$$

where $m$ denotes the iteration index. Note that due to the nonlinearity of this map, the solution of Eq. (15) with boundary conditions $\psi(0)=0$ and $\psi\left(Y_{0}\right)=\varphi / 2$ may be nonunique. To investigate this feature let us consider the streamfunction in (15) of the form

$$
\psi(z, r)=\Psi(y)+\varepsilon \tilde{\psi}(y) \exp (i \kappa z),
$$

where $\Psi(y)$ is the base flow given by $(17), \widetilde{\psi}(y)$ is a perturbation of the base flow (Fig. 18), and $\kappa$ is the axial wave number of this perturbation.

Introducing (19) in Eq. (15) one obtains the linearized equation

$$
\frac{d^{2} \tilde{\psi}}{d y^{2}}-\left(H^{\prime \prime}(\Psi)-\frac{K^{\prime 2}(\Psi)+K(\Psi) K^{\prime \prime}(\Psi)}{2 y}\right) \tilde{\psi}=\frac{\kappa^{2}}{2 y} \widetilde{\psi} .
$$

Of course, in order to preserve the flow rate the perturbation must satisfy homogeneous boundary conditions

$$
\tilde{\psi}(0)=\tilde{\psi}\left(Y_{0}\right)=0 .
$$

Equations (20) define a generalized eigenvalue problem. The eigenvalues $\kappa^{2}$ can be computed numerically once the problem is discretized. The expression inside square brackets on the left-hand side can be easily evaluated once an analytical swirl representation is available:

$$
C(y) \equiv H^{\prime \prime}(\Psi)-\frac{K^{\prime 2}(\Psi)+K(\Psi) K^{\prime \prime}(\Psi)}{2 y}=\frac{1}{u} \frac{d^{2} u}{d y^{2}}-\frac{K}{2 y^{2} u^{2}} \frac{d K}{d y} .
$$

If we consider a grid $y_{0}=0, y_{1}, \ldots, y_{1}, \ldots, y_{N}, y_{N+1}=Y_{0}$ and a piecewise linear approximation of the solution $\widetilde{\psi}(y) \approx \sum_{j} \tilde{\psi}_{j} N_{j}(y)$, the finite element discretization of problem (20) can be written in matrix form as

$$
\mathbf{A} \tilde{\psi}=\kappa^{2} \mathbf{B} \tilde{\psi}
$$

where $\tilde{\boldsymbol{\psi}}$ is the nodal values vector, and

$$
\begin{gathered}
A_{i j}=-\int \frac{d N_{i}(y)}{d y} \frac{d N_{j}(y)}{d y} d y-\int N_{i}(y) C(y) N_{j}(y) d y, \\
B_{i j}=\int \frac{N_{i}(y) N_{j}(y)}{2 y} d y
\end{gathered}
$$

are $N \times N$ tridiagonal symmetric matrices. Obviously the matrix entries in $(22 b)$ are evaluated only for the $N$ interior nodes, due to the homogeneous Dirichlet conditions $(20 b)$. The GVCSP procedure from the International Math and Statistics Libraries (IMSL) [26] is used here to compute all of the eigenvalues and eigenvectors of the generalized real symmetric eigenvalue problem $(22 a)$, with $\mathbf{B}$ symmetric and positive definite.

Let us summarize now the swirling flow model according to the synoptic Fig. 18. Once the analytical representation for axial and circumferential velocity components has been established, the mean flow streamfunction can be computed. A streamtube $\Psi$ $=$ const may be subject to axisymmetric perturbations $\varepsilon \widetilde{\psi}$, which are the eigenfunctions of problem (20). Such a perturbation can be

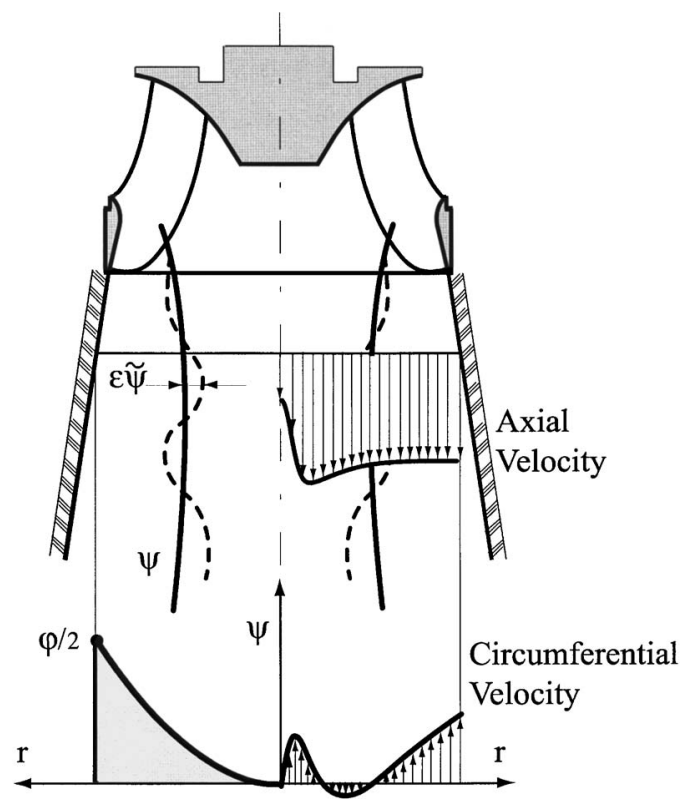

Fig. 18 Synoptic view of the model for swirling flow downstream of a Francis turbine runner 


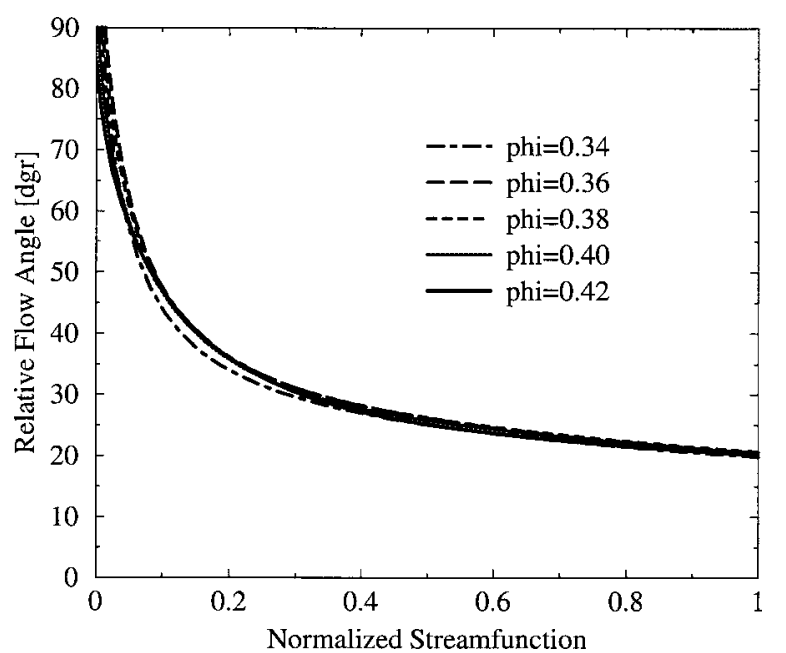

Fig. 19 Relative flow angle on streamtubes

sustained or not depending on the sign of the corresponding eigenvalue $\kappa^{2}$, according to the analysis to be presented in the next section.

\section{Analysis of the Swirling Flow}

An important property of the swirling flows downstream a constant pitch hydraulic turbine runner is that the relative flow angle depends only on the blade exit angle provided that the flow remains attached. This is certainly the case for a turbine runner operating in the neighborhood of the best efficiency point, therefore we can expect that the relative flow angle remains practically independent for the operating points of interest in this study. Moreover, due to negligible retarding forces, the global moment of momentum of the flow at the runner outlet should remain constant in the cone. Therefore, the relative flow angle (8) should depend only on the streamtube, i.e., on the normalized streamfunction $\psi /(\varphi / 2)$ (Fig. 19). In other words, the relative flow angle on the survey section at runner outlet is practically constant on a streamtube originating at the same radius on the blade trailing edge, being determined by the blade exit angle irrespective of the discharge. This shows the direct correlation between the runner blade design and the kinematics of the swirl on the draft tube inlet. Moreover, the significant changes in the circumferential and axial velocity profiles can be associated only with the streamtube's cross-section variation downstream the blade trailing edge, as the discharge is modified.

The above considerations on the relative flow angle (8) help us understand the striking feature that the flow rotates in some radius range in the opposite direction to that at smaller and larger radii. Since the relative flow angle remains constant on a streamtube, an increase in the dimensionless axial velocity $u$ must be accompanied by an increase in the dimensionless relative circumferential velocity $r$ - $w$. In consequence, as the axial flow accelerates, i.e., a jetlike axial velocity profile is developed when the discharge increases, the corresponding absolute circumferential velocity $w$ becomes negative in order to increase $r-w$, thus keeping $u /(r-w)$ practically constant in Eq. (8).

A global quantitative description of the swirling flow is provided by the swirl number $S$ defined as the axial flux of swirl momentum divided by the axial flux of axial momentum [[27], p. 2],

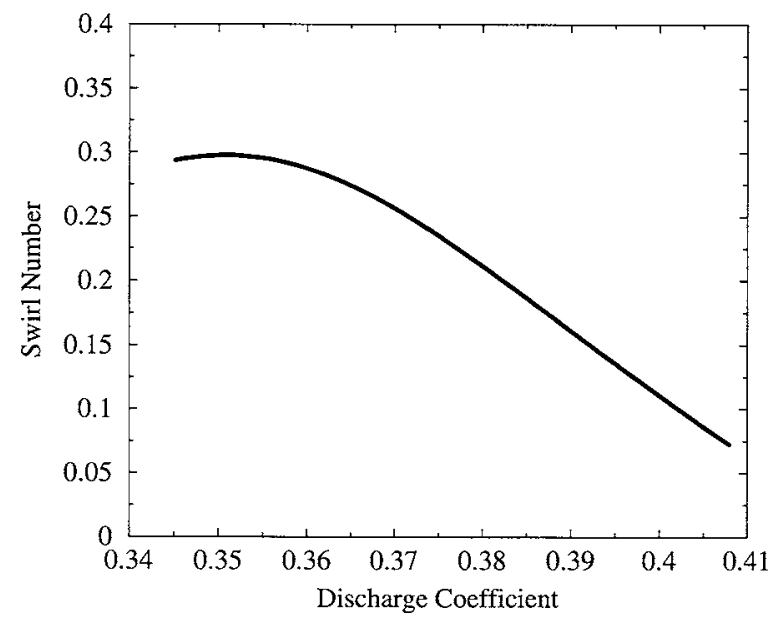

Fig. 20 The swirlnumber $S$ from (23a) versus the discharge coefficient $\varphi$

$$
S=\frac{\int_{0}^{R_{0}} \rho u(r w) r d r}{R_{0} \int_{0}^{R_{0}}\left(\rho u^{2}+p-p_{R_{0}}\right) r d r},
$$

where $p_{R_{0}}$ is the pressure at the wall. The pressure term in $(23 a)$ can be evaluated using the equation of radial equilibrium,

$$
\frac{\partial p}{\partial r}=\frac{\rho w^{2}}{r} \Rightarrow p-p_{R_{0}}=-\int_{r}^{R_{0}} \frac{\rho w^{2}}{r} d r .
$$

The swirl number computed for the swirling flow given by (9), with parameters from Figs. 15-17, is plotted versus the discharge coefficient in Fig. 20. One can see that for the investigated range of $\varphi$ the swirl number decreases as the discharge increases, but nevertheless the variation is smooth.

More interesting and useful conclusions can be drawn from the analysis of eigenvalues $\kappa^{2}$ and the corresponding eigenvectors in (20). If $\kappa^{2}<0$, then $\kappa$ is imaginary and the exponential factor in (19) will be $\exp ( \pm|\kappa| z)$. As we move downstream the current section, $z>0$, the only physically acceptable solution corresponds to $\exp (-|\kappa| z)$, showing an exponential damping of $\tilde{\psi}$. A swirl configuration for which all eigenvalues are negative is unable to sustain axisymmetric small-disturbance standing waves and it was termed supercritical by Benjamin [11]. On the other hand, if at least one eigenvalue $\kappa^{2}$ is positive, then the perturbation will take the form of a standing wave $\exp ( \pm i \kappa z)$, and the corresponding flow is termed subcritical. All physical interpretations attempted for the distinction between supercritical and subcritical states were mainly focused at the vortex breakdown phenomena. Benjamin's original interpretation was that for a given distribution of $H(\psi)$ and $K(\psi)$ one possible state of flow is subcritical and the conjugate state is supercritical. A deduction of this theory is that, compared with their conjugates, supercritical flows possess a deficiency of total momentum defined as the integral of axial momentum flux plus pressure over a cross section. This property would imply that supercritical flows are liable to undergo spontaneous transitions to subcritical state. Later, this theory came under quite heavy criticism, mostly because of its lack of explaining the axial flow reversal associated with the vortex breakdown. For example, Hall [28] particularly disagrees with the hydraulic jump analogy. Leibovich [29] considers that the most serious weakness of Benjamin's theory is that there is no clear way to relate it to experiments which, at high Reynolds numbers, always have unsteady, non-axisymmetric wakes. A decade ago Keller [12] 


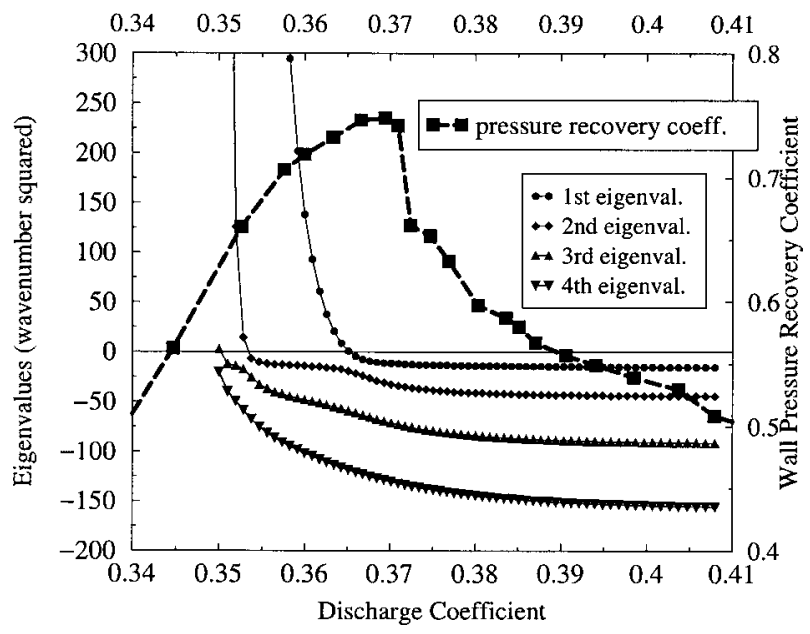

Fig. 21 The first four eigenvalues and the pressure recovery coefficient function of the discharge coefficient

pointed that various authors or even schools have conflicting views on the correct interpretation of the physics of vortex breakdown. Moreover, in his opinion, Benjamin's theory already contained most ideas for a successful theory of axisymmetric vortex breakdown but it was missing the definition of the total head circulation in regions with flow reversal, i.e., beyond the upstream interval of streamfunction values. The stagnation model emerged, where the total head is uniform and circulation vanishes in the domain of flow reversal, and was lately employed by Rusak et al. [30] to examine axisymmetric vortex breakdown in a finite length pipe. They present a comprehensive study of the Burgers vortex behavior, using both steady and unsteady axisymmetric inviscid flow models. Using essentially the same linearized eigenvalue problem, they determined the critical swirl level above which the base solution will evolve downstream to a solution that is a global (not local) minimizer of a certain functional. The flow in the pipe is computed explicitly, thus supporting the conclusions. However, no attempt has been made to directly correlate the computational results with any experimental data.

The approach we take in this paper is to examine the transition of the swirling flow downstream a Francis turbine runner from subcritical to supercritical as the discharge coefficient increases and to correlate the critical state with the experimentally observed sudden drop in the draft tube pressure recovery coefficient. A similar approach was advocated by Goldshtik and Hussain [14] who consider that vortex breakdown necessarily occurs when solution nonuniqueness is achieved by a continuous change in flow parameters. Moreover, we consider that valuable insight might be gained also by examining the eigenmodes $\widetilde{\psi}$ corresponding to positive eigenvalues.

Let us examine first the main result of this paper, inferred from Fig. 21. For $\varphi>0.365$, and correspondingly smaller swirl numbers, all eigenvalues from (20) are negative, thus the flow is supercritical and cannot sustain axisymmetric standing waves. However, for $\varphi<0.365$ the largest eigenvalue becomes positive, followed by the next eigenvalues as $\varphi$ decreases, and the flow is subcritical with standing waves described by the corresponding eigenvectors $\widetilde{\psi}$. The critical state occurs according to our computations at $\varphi=0.365$. This discharge value is quite close (only $1.3 \%$ smaller) to the value of $\varphi=0.37$ where the sudden drop in draft tube pressure recovery coefficient is observed. It seems reasonable to assume that the critical state is directly related to this experimentally observed phenomenon, since by trying several draft tube geometries while keeping the same runner (and the swirling flow) the same behavior has been observed practically at the same discharge. While reaching the critical swirl configuration seems to be

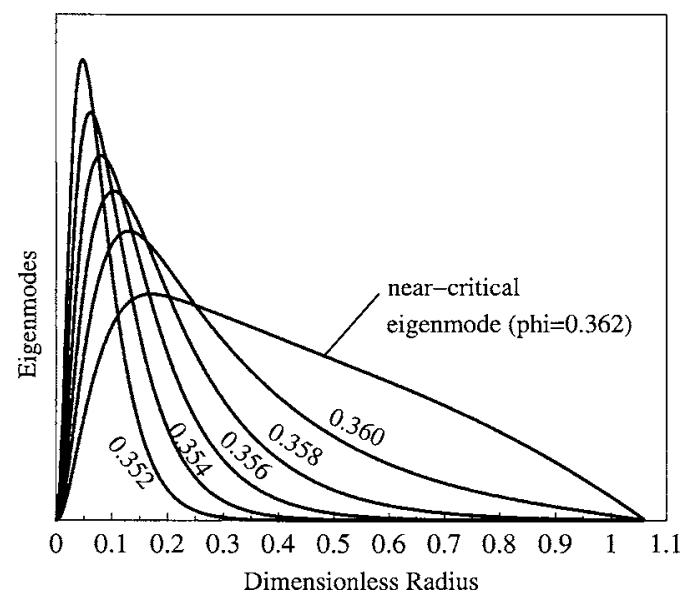

Fig. 22 Eigenmodes corresponding to the largest (positive) eigenvalue for subcritical swirling flows

the cause, the actual physical mechanism by which the pressure recovery suffers an abrupt change cannot be inferred from the present analysis. Experimental [2] as well as numerical $[3,4]$ investigations offer a comprehensive analysis of the Francis turbine draft tube flow.

Several eigenmodes $\tilde{\psi}(r)$ corresponding to the largest eigenvalue for subcritical flows are shown in Fig. 22. Since the eigenmodes are defined up to a multiplicative constant, it makes no sense to have marks on the vertical axis. One can easily see that as the discharge coefficient decreases the support of the eigenmode shrinks toward the axis neighborhood. It means that the induced velocity perturbations, for example their real part

$$
\tilde{u}=\frac{d \tilde{\psi}}{d y} \cos (\kappa z) \quad \text { and } \quad \tilde{v}=\frac{\kappa \tilde{\psi}}{\sqrt{2 y}} \sin (\kappa z),
$$

are confined closer to the axis as $\varphi$ decreases. Moreover, the relative amplitude of the perturbation increases since $\kappa$ gets larger (leading to larger $\widetilde{v}$ ) and also the slope at the origin increases (leading to larger $\tilde{u}$ ) on the axis. Although no vortex breakdown bubble is observed in the draft tube cone, the above velocity perturbations can be related to the axial velocity deficit reduction further downstream. This mechanism of reducing the "wakelike" axial velocity nonuniformity might be responsible for the improvement in the draft tube overall performance as the discharge gets smaller than the critical value. For even smaller discharge, more eigenmodes are successively present. For example, Fig. 23 presents the first two eigenmodes at $\varphi=0.348$, corresponding to the two positive eigenvalues. While the first eigenmode is confined near the axis, the second one is not, but its behavior will follow the same pattern when further decreasing the discharge.

\section{Conclusions}

The present work started from the idea that the swirling flow configuration at the outlet of a Francis turbine runner has a major influence on the overall behavior of the flow downstream in the draft tube.

We have investigated experimentally the velocity axial and circumferential components at the runner outlet for 17 operating points within the turbine normal operating range. Then, a suitable analytical representation of the velocity profiles is developed, with the turbine discharge as an independent parameter. It is shown that the swirling flow in the survey section can be accurately represented using a superposition of three distinct vortices: a rigid body rotation motion, a counter-rotating and co-flowing Batchelor vortex with large core radius, and a co-rotating and counter-flowing Batchelor vortex with small vortex core. The eight parameters of 


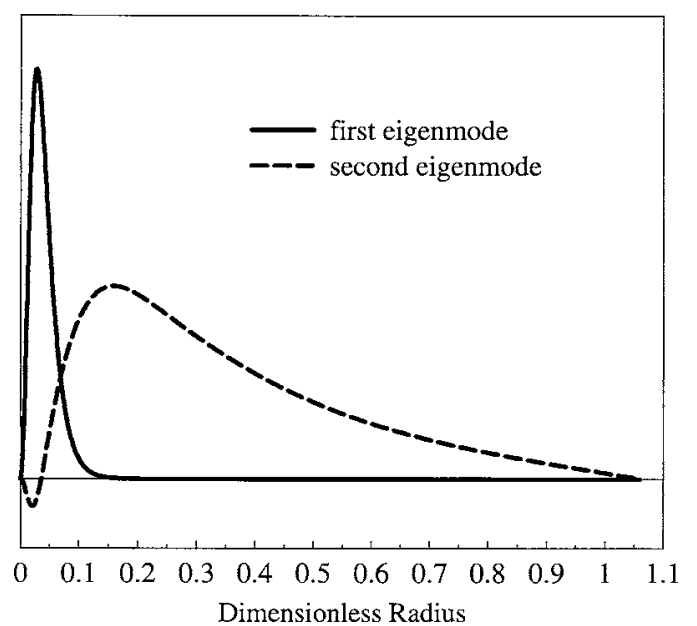

Fig. 23 First two eigenmodes for subcritical swirling flow at $\varphi=0.348$

this three-vortex system are determined by fitting the experimental data and are shown to vary smoothly (generally linear) with the discharge coefficient.

The flow at the runner outlet is then analyzed using the mathematical model for a steady, axisymmetric, and inviscid swirling flow. Following Benjamin's theory of finite transitions between frictionless cylindrical flows, we have performed an eigenvalue analysis of the linearized problem. It is shown that the swirl reaches a critical state at discharge $\varphi=0.365$. For larger discharge the flow ingested by the draft tube is supercritical, while at lower discharge it is subcritical. The critical state occurs quite close to the discharge $\varphi=0.370$ where a sudden variation in the draft tube pressure recovery, as well as in the overall turbine efficiency, is experimentally observed. For the particular turbine under investigation this discharge value happens to correspond to the best efficiency point, leading to a negative impact on the turbine regulation.

A qualitative correlation between the swirling flow at the draft tube inlet and the complex flow behavior further downstream may be inferred in conjunction with the Werlé-Legendre separation in the bend, discovered by Mauri et al. [15]. For subcritical swirling flow the sustained axisymmetric waves weaken the integrity of the vortex core, thus preventing the interaction with secondary flows in the draft tube bend. As the swirling flow reaches the critical state, and becomes supercritical as the discharge increases, the vortex core is no longer affected by axisymmetric perturbations, thus being able to trigger a global Werlé-Legendre separation that blocks the right channel of the draft tube and accelerates the flow in the other channel. The static pressure recovery is strongly affected, leading to an important loss in the overall machine efficiency.

Our analysis leads to the conclusion that when designing or optimizing turbine runners one should avoid reaching a critical state for the swirl at the runner outlet within the normal operating range.

\section{Acknowledgment}

The authors take this opportunity to thank our partners in the FLINDT Project Eureka No. 1625, Alstom Hydro, Electricité de France, GE Hydro, VA Tech Hydro, Voith-Siemens Hydro, PSEL (Funds for Projects and Studies of the Swiss Electric Utilities), and the CTI (Commission for Technology and Innovation), for their financial support. We also thank the staff of the Laboratory for Hydraulic Machines from the École Polytechnique Fédérale de Lausanne for their invaluable technical support. The first author would like to thank the École Polytechnique Fédérale de Lau- sanne, where he completed most of his contribution to this paper while a Visiting Professor in April 2003 and April-May 2004

\section{Nomenclature}

$A_{\text {ref }}=$ reference section area

$H=$ Bernoulli's total head

$K=$ circulation function

$Q=$ turbine discharge

$R_{0}=$ survey section radius

$R_{\text {ref }}=$ reference section radius

$R_{1}, R_{2}=$ vortex core radii

$S=$ swirl number

$U_{0}, U_{1}, U_{2}=$ vortex characteristic axial velocities

$p=$ pressure

$r=$ radial coordinate

$u, \tilde{u}=$ axial velocity and its perturbation

$v, \widetilde{v}=$ radial velocity and its perturbation

$w=$ circumferential velocity

$y=$ auxiliary variable

$z=$ axial coordinate, aligned with the vertical machine axis

$\Omega_{0}, \Omega_{1}, \Omega_{2}=$ vortex characteristic angular velocities

$\beta=$ relative flow angle

$\chi=$ draft tube wall pressure recovery coefficient

$\kappa=$ wave number

$\psi=$ streamfunction

$\varphi=Q / \omega R_{r e f} A_{r e f}$ discharge coefficient

$\rho=$ density

\section{References}

[1] Escudier, M., 1987, "Confined Vortices in Flow Machinery," Annu. Rev. Fluid Mech., 19, pp. 27-52.

[2] Avellan, F., 2000, "Flow Investigation in a Francis Draft Tube: the FLINDT Project," in Proceedings of the 20th IAHR Symposium, Charlotte, NC.

[3] Mauri, S., 2002, "Numerical Simulation and Flow Analysis of an Elbow Diffuser," Ph.D. thesis, École Politechnique Fédérale de Lausanne, Lausanne, Switzerland.

[4] Mauri, S., Kueny, J.-L., and Avellan, F., 2000, "Numerical Prediction of the Flow in a Turbine Draft Tube. Influence of the Boundary Conditions," FEDSM'00-11084, Proceedings of the ASME 2000 Fluids Engineering Division Summer Meeting, Boston, MA.

[5] Engström, T. F., 2003, "Simulation and Experiments of Turbulent Diffuser Flow With Hydropower Applications," Ph.D. thesis, Luleå University of Technology, Luleå, Sweden.

[6] Cervantes, M. J., 2003, "Effects of Boundary Conditions and Unsteadiness on Draft Tube Flow," Ph.D. thesis, Luleå University of Technology, Luleå, Sweden.

[7] Harvey, J. K., 1962, "Some Observations of the Vortex Breakdown Phenomenon,” J. Fluid Mech., 14, pp. 585-592.

[8] Faler, J. H., and Leibovich, S., 1977, "Disrupted States of Vortex Flow and Vortex Breakdown,” Phys. Fluids, 20(9), pp. 1385-1400.

[9] Mattner, T. W., Joubert, P. N., and Chong, M. S., 2002, "Vortical Flow. Part 1. Flow Through a Constant Diameter Pipe," J. Fluid Mech., 463, pp. 259-291.

[10] Snyder, D. O., and Spall, R. E., 2000, "Numerical Simulation of Bubble-Type Vortex Breakdown Within a Tube-and-Vane Apparatus," Phys. Fluids, 12(3), pp. 603-608.

[11] Benjamin, T. J., 1962, "Theory of the Vortex Breakdown Phenomenon," J. Fluid Mech., 14, pp. 593-629.

[12] Keller, J. J., 1995, "On the Interpretation of Vortex Breakdown," Phys. Fluids, 7(7), pp. 1695-1702.

[13] Leibovich, S., 1978, "The Structure of Vortex Breakdown," Annu. Rev. Fluid Mech., 10, pp. 221-246.

[14] Goldshtik, M., and Hussain, F., 1998, "Analysis of Inviscid Vortex Breakdown in a Semi-infinite Pipe,” Fluid Dyn. Res., 23, pp. 189-234.

[15] Mauri, S., Kueny, J.-L., and Avellan, F., 2004, "Werlé-Legendre Separation in a Hydraulic Machine Draft Tube," ASME J. Fluids Eng., 126, pp. 976-980.

[16] International Electrotechnical Commision, 1999, "Hydraulic Turbines, Storage Pumps and Pump-Turbines-Model Acceptance Tests," International Standard IEC 60193, 2nd ed.

[17] Ciocan, G. D., 1998, “Contribution à l'Analyse des Ecoulements 3D Complexes en Turbomachines," Ph.D. thesis, Institut National Polytechnique de Grenoble, Grenoble, France.

[18] Ciocan, G. D., Avellan, F., and Kueny, J.-L., 2000, "Optical Measurement Techniques for Experimental Analysis of Hydraulic Turbines Rotor-Stator Interaction," FEDSM2000-11056, Proceedings of the ASME 2000 Fluids Engineering Division Summer Meeting, Boston, MA.

[19] Batchelor, G. K., 1964, "Axial Flow in Trailing Line Vortices," J. Fluid Mech., 
20(4), pp. 645-658.

[20] Alekseenko, S. V., Kuibin, P. A., Okulov, V. L., and Shtork, S. I., 1999 "Helical Vortices in Swirl Flow," J. Fluid Mech., 382, pp. 195-243.

[21] Wang, S., and Rusak, A., 1997, "The Dynamics of a Swirling Flow in a Pipe and Transition to Axisymmetric Vortex Breakdown," J. Fluid Mech., 340, pp. $177-223$.

[22] Lu, P., and Semião, V., 2003, "A New Second-Moment Closure Approach for Turbulent Swirling Confined Flow," Int. J. Numer. Methods Fluids, 41, pp. $133-150$.

[23] Shtern, V., and Hussain, F., 2003, "Effect of Deceleration on Jet Instability," J. Fluid Mech., 480, pp. 283-309.

[24] Buntine, J. D., and Saffman, P. G., 1995, "Inviscid Swirling Flows and Vortex Breakdown," Proc. R. Soc. London, Ser. A, 449, pp. 139-153.
[25] Tsai, C.-Y., 1980, "Examination of Group-Velocity Criterion for Breakdown of Vortex Flow in a Divergent Duct," Phys. Fluids, 23(5), pp. 864-870.

[26] Visual Numerics, 2003, "IMSL Fortran Library User's Guide. Mathematical Functions in Fortran."

[27] Gupta, A. K., Lilley, D. G., and Syred, N., 1984, Swirl Flows, Abacus Press, Cambridge, MA.

[28] Hall, M. G., 1972, "Vortex Breakdown,” Annu. Rev. Fluid Mech., 4, pp. 195-218.

[29] Leibovich, S., 1984, "Vortex Stability and Breakdown: Survey and Extension," AIAA J., 22(9), pp. 1192-1206.

[30] Rusak, Z., Wang, S., and Whiting, C. H., 1998, "The Evolution of a Perturbed Vortex in a Pipe to Axisymmetric Vortex Breakdown," J. Fluid Mech., 366, pp. 211-237. 